\title{
SURVEY ARTICLE: CONTINUED FRACTIONS ASSOCIATED WITH WIENER-LEVINSON FILTERS, FREQUENCY ANALYSIS, MOMENT THEORY AND POLYNOMIALS ORTHOGONAL ON THE UNIT CIRCLE
}

\author{
C. BONAN-HAMADA, W.B. JONES AND O. NJÅSTAD
}

\begin{abstract}
This paper surveys the close relationships among the topics included in the title. Emphasis is given to the family of positive Perron-Carathéodory continued fractions (PPC-fractions) which play a central role in the theory of trigonometric moment problems and Szegö polynomials orthogonal on the unit circle. An important application of PPC-fractions is frequency analysis of discrete time signals using Wiener-Levinson digital filters with illustrations given from computational experiments.
\end{abstract}

1. Introduction. Continued fractions have played a fundamental role in the origin and development of moment theory and orthogonal polynomials. The classical Stieltjes moment problem, posed and solved in the celebrated memoir [71], made essential use of Stieltjes continued fractions

$$
\frac{a_{1} z}{1}+\frac{a_{2} z}{1}+\frac{a_{3} z}{1}+\cdots, \quad a_{n}>0, n=1,2,3, \ldots,
$$

where $z$ is a complex variable. The first full treatment of the classical Hamburger moment problem [22] was based on real J-fractions

$$
\frac{a_{1}}{b_{1}+z}-\frac{a_{2}}{b_{2}+z}-\frac{a_{3}}{b_{3}+z}-\cdots
$$

where

$$
a_{n}>0, \quad b_{n} \in \mathbb{R}, \quad n=1,2,3, \ldots .
$$

2010 AMS Mathematics subject classification. Primary 30B70, Secondary 30E05, 33C45, 40A15, 42A70, 42C05, 44A60, 93E11.

Keywords and phrases. Continued fractions, Wiener-Levinson filters, frequency analysis, moment theory, orthogonal polynomials.

Received by the editors on March 9, 2015, and in revised form on November 5, 2015. 
It is well documented that the study of orthogonal polynomials originated in the theory of continued fractions (1.2) [72, page 54]. Other moment theory and orthogonal functions investigated by means of continued fractions include the strong Stieltjes moment problem [45], the strong Hamburger moment problem [30, 31] and orthogonal Laurent polynomials $[31,42,54]$.

A family of continued fractions, called positive Perron-Carathéodory fractions (PPC-fractions) plays a role for the trigonometric moment problem and Szegö polynomials that is analogous to that of the continued fractions (1.1) and (1.2) for classical moment problems and orthogonal polynomials. The PPC-fractions, introduced in $[\mathbf{3 2}, \mathbf{3 3}, \mathbf{3 4}]$, have the form

$$
\delta_{0}-\frac{2 \delta_{0}}{1}+\frac{1}{\overline{\delta_{1}} z}+\frac{\left(1-\left|\delta_{1}\right|^{2}\right) z}{\delta_{1}}+\frac{1}{\overline{\delta_{2}} z}+\frac{\left(1-\left|\delta_{2}\right|^{2}\right) z}{\delta_{2}}+\cdots
$$

where

$$
\delta_{0}>0, \quad \delta_{n} \in \mathbb{C}, \quad\left|\delta_{n}\right|<1, \quad n=1,2,3, \ldots
$$

An important application of PPC-fractions and their denominators of odd order (the Szegö polynomials) is frequency analysis based on Wiener-Levinson digital filters $[\mathbf{5}, \mathbf{2 0}, \mathbf{4 7}, \mathbf{5 2}, \mathbf{6 6}, \mathbf{7 6}]$. Frequency analysis is the determination of unknown frequencies in a discrete time signal consisting of a superposition of sinusoidal waves. Speech processing and other applications in real time are made possible by fast computational methods such as Levinson's algorithm for computing the reflection coefficients $\delta_{n}$ of the associated PPC-fraction (1.3) and related algorithms for solving Toeplitz systems of equations $[\mathbf{3}, \mathbf{4}, \mathbf{6}$, $10,11,14,15,16,26]$. Theoretical foundations for these applications using PPC-fractions and Szegö polynomials are given in a series of papers $[28,29,35,36,39,40,48,53,55,56,57,58,69]$. Some illustrations from computational experiments are given in Section 9 of this paper and also in $[37,38,41]$.

The study of PPC-fractions and their applications has been published in a large number of papers, many of which are not readily accessible. The purpose of the present article is to provide a unified, concise and self-contained survey of this work, giving proofs that are attainable without excessive effort. For the most part, proofs are based 
on elementary and constructive methods; some provide estimates of the speed of convergence and truncation error bounds.

Equations (1.4)-(1.9) provide some basic notation, definitions and formulas in continued fraction theory. See, e.g., $[\mathbf{9}, \mathbf{2 3}, \mathbf{4 2}, \mathbf{4 9}, \mathbf{5 0}$, $\mathbf{5 9}, \mathbf{7 5}]$. We use the standard notation for continued fractions

$$
b_{0}+\frac{a_{1}}{b_{1}}+\frac{a_{2}}{b_{2}}+\frac{a_{3}}{b_{3}}+\cdots=b_{0}+\frac{a_{1}}{b_{1}+\frac{a_{2}}{b_{2}+\frac{a_{3}}{b_{3}+\frac{a_{4}}{\ddots}}}},
$$

where

$$
0 \neq a_{n} \in \mathbb{C}, \quad b_{n} \in \mathbb{C}, \quad n=0,1,2, \ldots
$$

The $n$th numerator $A_{n}$ and nth denominator $B_{n}$ of the continued fraction (1.4) are defined by the difference equations

$$
\begin{aligned}
& A_{-1}=1, \quad B_{-1}=0, \quad A_{0}=b_{0}, \quad B_{0}=1 \\
& \left(\begin{array}{l}
A_{n} \\
B_{n}
\end{array}\right)=b_{n}\left(\begin{array}{l}
A_{n-1} \\
B_{n-1}
\end{array}\right)+a_{n}\left(\begin{array}{l}
A_{n-2} \\
B_{n-2}
\end{array}\right), \quad n=1,2,3, \ldots
\end{aligned}
$$

They satisfy the determinant formulas:

$$
A_{n} B_{n-1}-A_{n-1} B_{n}=(-1)^{n-1} \prod_{j=1}^{n} a_{j}, \quad n=1,2,3, \ldots
$$

The associated linear fractional transformations

$$
\begin{aligned}
& \text { (1.7a) } \quad s_{0}(\omega)=b_{0}+\omega, \quad s_{n}(\omega)=\frac{a_{n}}{b_{n}+\omega}, \quad n=1,2,3, \ldots, \\
& (1.7 \mathrm{~b}) S_{0}(\omega)=s_{0}(\omega), \quad S_{n}(\omega)=S_{n-1}\left(s_{n}(\omega)\right), \quad n=1,2,3, \ldots,
\end{aligned}
$$

provide the useful relationships

$$
\begin{aligned}
S_{n}(\omega)= & \frac{A_{n}+A_{n-1} \omega}{B_{n}+B_{n-1} \omega}=b_{0}+\frac{a_{1}}{b_{1}}+\frac{a_{2}}{b_{2}}+\cdots \\
& +\frac{a_{n-1}}{b_{n-1}}+\frac{a_{n}}{b_{n}+\omega} .
\end{aligned}
$$


Therefore, the $n$th approximant $S_{n}(0)$ of the continued fraction (1.4) is given by

$$
S_{n}(0)=\frac{A_{n}}{B_{n}}=b_{0}+\frac{a_{1}}{b_{1}}+\frac{a_{2}}{b_{2}}+\cdots+\frac{a_{n}}{b_{n}}, \quad n=0,1,2, \ldots
$$

From the difference equations (1.5), it follows that for PPC-fractions (1.3), the $n$th numerator $P_{n}(z)$ and $n$th denominator $Q_{n}(z)$ are defined by

$$
P_{0}(z)=\delta_{0}, \quad Q_{0}(z)=1, \quad P_{1}(z)=-\delta_{0}, \quad Q_{1}(z)=1
$$

and for $n=1,2,3, \ldots$,

$$
\left(\begin{array}{c}
P_{2 n}(z) \\
Q_{2 n}(z)
\end{array}\right)=\bar{\delta}_{n} z\left(\begin{array}{c}
P_{2 n-1}(z) \\
Q_{2 n-1}(z)
\end{array}\right)+\left(\begin{array}{c}
P_{2 n-2}(z) \\
Q_{2 n-2}(z)
\end{array}\right)
$$

and

$$
\left(\begin{array}{c}
P_{2 n+1}(z) \\
Q_{2 n+1}(z)
\end{array}\right)=\delta_{n}\left(\begin{array}{c}
P_{2 n}(z) \\
Q_{2 n}(z)
\end{array}\right)+\left(1-\left|\delta_{n}\right|^{2}\right) z\left(\begin{array}{c}
P_{2 n-1}(z) \\
Q_{2 n-1}(z)
\end{array}\right)
$$

Hence, $P_{n}(z)$ and $Q_{n}(z)$ are polynomials in $z$ of the form (1.11a)

$$
P_{2 n}(z)=\sum_{j=0}^{n} p_{2 n, j} z^{j}, \quad Q_{2 n}(z)=\sum_{j=0}^{n} q_{2 n, j} z^{j}, \quad n=0,1,2, \ldots,
$$

$$
P_{2 n+1}(z)=\sum_{j=0}^{n} p_{2 n+1, j} z^{j}, \quad Q_{2 n+1}(z)=\sum_{j=0}^{n} q_{2 n+1, j} z^{j}, \quad n=0,1,2, \ldots,
$$

where $p_{m, j} \in \mathbb{C}, q_{m, j} \in \mathbb{C}$, for $m=0,1,2, \ldots$, and

(1.11c) $\quad p_{2 n, 0}=\delta_{0}, \quad q_{2 n, 0}=1, \quad p_{2 n+1, n}=-\delta_{0}, \quad q_{2 n+1, n}=1$.

Thus, the $(2 n)$ th approximant $f_{n}(z)=P_{2 n}(z) / Q_{2 n}(z)$ is a rational function holomorphic at $z=0$, and the $(2 n+1)$ th approximant $g_{n}(z)=P_{2 n+1}(z) / Q_{2 n+1}(z)$ is a rational function holomorphic at $z=\infty$. It is shown (Section 2) that $\left\{f_{n}(z)\right\}_{n=0}^{\infty}$ converges to a function $f(z)$ holomorphic in the disk $|z|<1$ that satisfies

$$
\operatorname{Re} f(z)>0 \text { for }|z|<1, f(0)>0 \text {. }
$$


Moreover, there exists a sequence $\left\{\mu_{n}\right\}_{n=0}^{\infty}$ in $\mathbb{C}$ such that

$$
f(z)=\mu_{0}+2 \sum_{k=1}^{\infty} \mu_{k} z^{k}, \quad|z|<1,
$$

and, for each integer $n \geq 0$, there exist coefficients $\mu_{k}^{(n)} \in \mathbb{C}$ such that

$$
f_{n}(z)=\mu_{0}+2 \sum_{k=1}^{n} \mu_{k} z^{k}+\sum_{k=n+1}^{\infty} \mu_{k}^{(n)} z^{k},
$$

for all $z$ in a neighborhood of $z=0$. Thus, the coefficients of individual powers of $z$ in equation (1.13b) agree with those in equation (1.13a) for $0 \leq k \leq n, n \geq 0$. An analogous property holds for the sequence $\left\{g_{n}(z)\right\}_{n=0}^{\infty}$ of odd order approximants of the PPC fraction (1.3). This remarkable property of PPC-fractions is of great value and is exploited both for moment theory and for Szegö polynomials.

It is also useful to consider $M$-terminating PPC-fractions

$$
\begin{aligned}
\delta_{0}-\frac{2 \delta_{0}}{1}+\frac{1}{\overline{\delta_{1}} z}+\frac{\left(1-\left|\delta_{1}\right|^{2}\right) z}{\delta_{1}}+\cdots \\
+\frac{1}{\overline{\delta_{M-1}} z}+\frac{\left(1-\left|\delta_{M-1}\right|^{2}\right) z}{\delta_{M-1}}+\frac{1}{\overline{\delta_{M}} z},
\end{aligned}
$$

where $M$ is a positive integer and the complex coefficients $\delta_{n}$ satisfy

$$
\delta_{0}>0, \quad\left|\delta_{n}\right|<1 \quad \text { for } n=1,2, \ldots, M-1 \text { and }\left|\delta_{M}\right|=1 .
$$

For $0 \leq n \leq M$, the $n$th numerator $P_{n}(z), n$th denominator $Q_{n}(z)$ and $n$th approximant $S_{n}(0)$ of the continued fraction (1.4) are defined by equations (1.10) and $S_{n}(0)=P_{n}(z) / Q_{n}(z)$. The $M$-terminating PPC-fraction (1.14) is said to represent the rational function

$$
f(z)=\frac{P_{2 M}(z)}{Q_{2 M}(z)} .
$$

By a distribution function on $[-\pi, \pi]$ is meant a real valued, bounded non-decreasing function $\psi(\theta)$ defined on $[-\pi, \pi]=\{\theta:-\pi \leq \theta \leq \pi\}$. The set of all distribution functions on $[-\pi, \pi]$ is denoted by $\Psi(-\pi, \pi)$. We consider the following sets:

$\Psi_{\infty}(-\pi, \pi)=\{\psi \in \Psi(-\pi, \pi): \psi$ has infinitely many points of increase $\}$, 


$$
\Psi_{M}(-\pi, \pi)=\{\psi \in \Psi(-\pi, \pi): \psi \quad \text { has } M \text { points of increase }\},
$$

where $M$ is a positive integer. The trigonometric moment problem (TMP) for a doubly infinite sequence $\left\{\mu_{n}\right\}_{-\infty}^{\infty}$ in $\mathbb{C}$ consists of finding necessary and sufficient conditions for the existence of a $\psi \in$ $\Psi_{\infty}(-\pi, \pi)$, such that

$$
\mu_{n}=\frac{1}{2 \pi} \int_{-\pi}^{\pi} e^{-i n \theta} d \psi(\theta), \quad n=0, \pm 1, \pm 2, \ldots
$$

Such a function $\psi$ is called a solution to the TMP. It is readily shown [1, Theorem 5.1.2] that, if a solution exists, then it is essentially unique. The number $\mu_{n}$ is called the nth moment with respect to $\psi$. Akhiezer and Krein [2] were the first to investigate trigonometric moment problems. Extensive expositions of the TMP can be found in the books $[1,17,18,19,21,46,70]$.

The approach for moment theory in the present paper establishes a one-to-one correspondence between PPC-fractions (1.3) and distribution functions $\psi \in \Psi_{\infty}(-\pi, \pi)$. Use is made of the connections (established by Toeplitz in [73]) between positive definite quadratic (Toeplitz) forms

$$
\sum_{j=-n}^{n} \sum_{k=-n}^{n} a_{j} \bar{a}_{k} \mu_{j-k}, \quad a_{j}, a_{k} \in \mathbb{C}
$$

and Toeplitz determinants $T_{k}^{(m)}$ associated with the sequence $\left\{\mu_{n}\right\}_{-\infty}^{\infty}$ where, for $m=0, \pm 1, \pm 2, \ldots$ and $k=1,2,3, \ldots$,

$$
T_{0}^{(m)}=1
$$

and

$$
T_{k}^{(m)}=\left|\begin{array}{cccc}
\mu_{m} & \mu_{m-1} & \cdots & \mu_{m-k+1} \\
\mu_{m+1} & \mu_{m} & \cdots & \mu_{m-k+2} \\
\vdots & \vdots & & \vdots \\
\mu_{m+k-1} & \mu_{m+k-2} & \cdots & \mu_{m}
\end{array}\right|
$$

It is shown (Theorem 2.2) that there exists a PPC-fraction whose sequence of even order approximants converges to a function $f(z)$ represented by the power series (1.13a) if and only if $\left\{\mu_{n}\right\}_{-\infty}^{\infty}$ satisfies

$$
\mu_{n}=\bar{\mu}_{-n} \quad \text { and } \quad T_{n}^{(0)}>0 \text { for } n=0,1,2, \ldots
$$


Conditions (1.18) are necessary and sufficient for existence of a solution to the TMP for $\left\{\mu_{n}\right\}_{-\infty}^{\infty}$. See Theorem 3.1.

Functions belonging to the class $\mathcal{C}$ defined by

$$
\begin{aligned}
\mathcal{C}=\{ & f: f(z) \text { is holomorphic in }|z|<1, f(0)>0 \text { and } \\
& \operatorname{Re} f(z)>0 \text { for }|z|<1\}
\end{aligned}
$$

are closely related to PPC-fractions and play an important role in trigonometric moment theory. The functions in $\mathcal{C}$ were introduced in $[7,8]$ and are called normalized Carathéodory functions. The HerglotzRiesz representation theorem $([\mathbf{1}, \mathbf{2 4}, \mathbf{6 7}, \mathbf{6 8}])$ asserts that for every $f \in \mathcal{C}$ there exists a $\psi \in \Psi(-\pi, \pi)$ such that

$$
f(z)=\frac{1}{2 \pi} \int_{-\pi}^{\pi} \frac{e^{i \theta}+z}{e^{i \theta}-z} d \psi(\theta), \quad|z|<1 .
$$

It is shown (Section 6) that, if $\psi \in \Psi_{\infty}(-\pi, \pi)$, then $f(z)$ is the limit of the even order approximants of a PPC-fraction and, if $\psi \in \Psi_{M}(-\pi, \pi)$, then $f(z)$ is represented by an M-terminating PPCfraction. Therefore, the class $\mathcal{C}$ of functions is completely characterized by PPC-fractions and M-terminating PPC-fractions.

Polynomials orthogonal on the unit circle were introduced by Szegö [72] using inner products with respect to a distribution function $\psi \in$ $\Psi_{\infty}(-\pi, \pi)$

$$
\langle P, Q\rangle_{\psi}=\frac{1}{2 \pi} \int_{-\pi}^{\pi} P\left(e^{i \theta}\right) \overline{Q\left(e^{i \theta}\right)} d \psi(\theta), \quad P, Q \in \Lambda
$$

where $\Lambda$ is the linear space

$$
\Lambda=\left\{\sum_{k=p}^{q} c_{k} z^{k}: c_{k} \in \mathbb{C}, p \leq q\right\} .
$$

If $Q_{n}(z)$ is the $n$th denominator of the PPC-fraction corresponding to $\psi$, then the nth degree, monic Szegö polynomial (orthogonal with respect to $\psi$ ) is given by

$$
\rho_{n}(z)=Q_{2 n+1}(z), \quad n=0,1,2, \ldots,
$$

and the $n$th reciprocal polynomial $\rho_{n}^{*}(z)=z^{n} \overline{\rho_{n}(1 / \bar{z})}$ is given by

$$
\rho_{n}^{*}(z)=Q_{2 n}(z), \quad n=0,1,2, \ldots
$$


Since Szegö polynomials are denominators of PPC-fractions, many of their properties given in Section 4 are immediate consequences of results on PPC-fractions established in Section 2. This work is essential for the study of frequency analysis based on Wiener-Levinson filters (Sections 7 and 8).

Let $B(t)$ be a real valued function of the form

$$
B(t)=\sum_{j=-I}^{I} \alpha_{j} e^{2 \pi i f_{j} t}, \quad t \in \mathbb{R}, I \in\{1,2,3, \ldots\},
$$

where the frequencies $f_{j}$ satisfy

$$
0=f_{0}<f_{1}<f_{2}<\cdots<f_{I}, \quad f_{j}=-f_{-j}, j=1,2, \ldots, I,
$$

and the amplitudes $\alpha_{j}$ satisfy

$$
\alpha_{0}>0, \quad 0 \neq \alpha_{j}=\overline{\alpha_{-j}} \in \mathbb{C}, \quad j=1,2, \ldots, I .
$$

Frequency analysis consists of determining the unknown frequencies $f_{j}$ using as input a finite sample of $N$ (observed) values

$$
\chi_{N}(m)=B\left(t_{m}\right)=\sum_{j=-I}^{I} \alpha_{j} e^{i \omega_{j} m}, \quad m=0,1,2, \ldots, N-1
$$

where the $\omega_{j}$ are normalized frequencies defined by

$$
\omega_{j}=2 \pi \Delta t f_{j}, \quad j=0, \pm 1, \pm 2, \ldots, \pm I
$$

and

$$
0<\Delta t<1 /\left(2 f_{I}\right)
$$

Frequency analysis based on Wiener-Levinson filters (Sections 7 and 8) uses a discrete time signal of the form (1.25) to construct Szegö polynomials $\rho_{n}\left(\psi_{N} ; z\right)$ with the property that, as $N \rightarrow \infty$, the zeros of $\rho_{n}\left(\psi_{N} ; z\right)$ with greatest moduli converge to the critical points $e^{i \omega_{j}}, j=0, \pm 1, \pm 2, \ldots, \pm I$ on the unit circle. Here, $\psi_{N}(\theta)$ is a distribution function defined by the signal $\left\{\chi_{N}(m)\right\}_{m=0}^{N-1}$. Wiener filters [76] were developed in the context of continuous time signals. The modification for discrete signals is the work of Levinson [47]. In the special case that there exists a frequency $f$ such that $f_{j}=j f$, $j=0,1, \ldots, I$, then the series (1.24) reduces to an ordinary Fourier 
series and $f$ is the fundamental harmonic. For speech processing and many other applications, the unknown frequencies $f_{j}$ are not multiples of a fundamental frequency, see $[\mathbf{1 6}, \mathbf{5 2}, \mathbf{6 6}]$.

2. Positive Perron-Carathéodory continued fractions. PPCfractions provide the structural framework for developing moment theory and orthogonal polynomials on the unit circle. From the difference equations (1.10) the $n$th numerator $P_{n}(z)$ and $n$th denominator $Q_{n}(z)$ satisfy, for $n=0,1,2, \ldots$,

$$
\begin{array}{ll}
P_{2 n}(z)=-z^{n} \overline{P_{2 n+1}(1 / \bar{z})}, & Q_{2 n}(z)=z^{n} \overline{Q_{2 n+1}(1 / \bar{z})}, \\
P_{2 n+1}(z)=-z^{n} \overline{P_{2 n}(1 / \bar{z})}, & Q_{2 n+1}(z)=z^{n} \overline{Q_{2 n}(1 / \bar{z})} .
\end{array}
$$

Also,

$$
f_{n}(z)=-\overline{g_{n}(1 / \bar{z})} \quad \text { and } \quad g_{n}(z)=-\overline{f_{n}(1 / \bar{z})}
$$

where

$$
f_{n}(z)=\frac{P_{2 n}(z)}{Q_{2 n}(z)} \quad \text { and } \quad g_{n}(z)=\frac{P_{2 n+1}(z)}{Q_{2 n+1}(z)} .
$$

It follows from the determinant formulas (1.6) that, for $n=1,2,3, \ldots$,

$$
P_{2 n}(z) Q_{2 n-1}(z)-P_{2 n-1}(z) Q_{2 n}(z)=2 \delta_{0} \prod_{j=1}^{n-1}\left(1-\left|\delta_{j}\right|^{2}\right) z^{n-1},
$$

and

$$
P_{2 n+1}(z) Q_{2 n}(z)-P_{2 n}(z) Q_{2 n+1}(z)=-2 \delta_{0} \prod_{j=1}^{n}\left(1-\left|\delta_{j}\right|^{2}\right) z^{n}
$$

For $n=0,1,2, \ldots$,

$$
P_{2 n+2}(z) Q_{2 n}(z)-P_{2 n}(z) Q_{2 n+2}(z)=-2 \delta_{0} \bar{\delta}_{n+1} \prod_{j=1}^{n}\left(1-\left|\delta_{j}\right|^{2}\right) z^{n+1},
$$

and

$$
P_{2 n+3}(z) Q_{2 n+1}(z)-P_{2 n+1}(z) Q_{2 n+3}(z)=2 \delta_{0} \delta_{n+1} \prod_{j=1}^{n}\left(1-\left|\delta_{j}\right|^{2}\right) z^{n+1} \text {. }
$$


In equation $(2.3 \mathrm{a})$ when $n=1$, the empty product is 1 by definition.

Since $P_{2 n}(z) / Q_{2 n}(z)$ is holomorphic at $z=0$ and $P_{2 n+1}(z) / Q_{2 n+1}(z)$ is holomorphic at $z=\infty$, we can express these functions as convergent power series

$$
\frac{P_{2 n}(z)}{Q_{2 n}(z)}=\mu_{0}^{(n)}+2 \sum_{k=1}^{\infty} \mu_{k}^{(n)} z^{k}, \quad \mu_{k}^{(n)} \in \mathbb{C},
$$

for all $z$ in a neighborhood of $z=0$, and

$$
\frac{P_{2 n+1}(z)}{Q_{2 n+1}(z)}=-\mu_{0}^{(n)}-2 \sum_{k=1}^{\infty} \mu_{-k}^{(n)} z^{-k}, \quad \mu_{-k}^{(n)} \in \mathbb{C},
$$

for all $z$ in a neighborhood of $z=\infty$. Dividing both sides of $(2.3 \mathrm{c})$ by the product $Q_{2 n}(z) Q_{2 n+2}(z)$ and dividing both sides of $(2.3 \mathrm{~d})$ by $Q_{2 n+1}(z) Q_{2 n+3}(z)$ yields the following theorem from [32]:

Theorem 2.1. Corresponding to each PPC-fraction (1.3), there exists a unique pair $\left(L_{0}, L_{\infty}\right)$ of formal power series (fps) of the form

$$
L_{0}=\mu_{0}+2 \sum_{k=1}^{\infty} \mu_{k} z^{k}, \quad L_{\infty}=-\mu_{0}-2 \sum_{k=1}^{\infty} \mu_{-k} z^{-k}, \quad \mu_{k} \in \mathbb{C},
$$

such that, for $n=0,1,2, \ldots$,

$$
L_{0}-\frac{P_{2 n}(z)}{Q_{2 n}(z)}=-2 \delta_{0} \prod_{j=1}^{n}\left(1-\left|\delta_{j}\right|^{2}\right) z^{n+1}+O\left(z^{n+2}\right),
$$

and

$$
L_{\infty}-\frac{P_{2 n+1}(z)}{Q_{2 n+1}(z)}=2 \delta_{0} \prod_{j=1}^{n}\left(1-\left|\delta_{j}\right|^{2}\right)\left(\frac{1}{z}\right)^{n+1}+O\left(\left(\frac{1}{z}\right)^{n+2}\right)
$$

It follows from equation (2.4) and Theorem 2.1 that, for $n=$ $0,1,2, \ldots$,

$$
\mu_{k}^{(n)}=\mu_{k}, \quad k=0, \pm 1, \pm 2, \ldots, \pm n .
$$

This is a remarkable and useful property of PPC-fractions in view of the fact (Theorem 2.3) that the rational functions (2.4a) and (2.4b) converge to functions $f(z)$ and $g(z)$, respectively. Theorem 2.1 not only 
enables us to establish the existence of the corresponding pair $\left(L_{0}, L_{\infty}\right)$ of series (2.5) but, in view of equation (2.7), we obtain explicit formulas for the PPC-fraction coefficients $\delta_{n}$ and the polynomials $Q_{n}(z)$ in terms of Toeplitz determinants (1.18).

\section{Theorem $2.2([32])$.}

(A) For a PPC-fraction (1.3), let $\left(L_{0}, L_{\infty}\right)$ be the corresponding pair of formal power series (2.5), and let $T_{k}^{(m)}$ denote the Toeplitz determinants (1.17) for the double sequence $\left\{\mu_{n}\right\}_{-\infty}^{\infty}$. Then, for $n=$ $1,2,3, \ldots$,

$$
\begin{gathered}
\mu_{0}=\delta_{0}>0, \quad \mu_{n}=\overline{\mu_{-n}}, \quad T_{n}^{(0)}>0, \\
\delta_{n}=(-1)^{n} \frac{T_{n}^{(-1)}}{T_{n}^{(0)}}, \quad \overline{\delta_{n}}=(-1)^{n} \frac{T_{n}^{(1)}}{T_{n}^{(0)}}, \\
1-\left|\delta_{n}\right|^{2}=\frac{T_{n+1}^{(0)} T_{n-1}^{(0)}}{\left(T_{n}^{(0)}\right)^{2}},
\end{gathered}
$$

$$
\begin{array}{r}
Q_{2 n}(z)=\frac{1}{T_{n}^{(0)}}\left|\begin{array}{cccc}
\mu_{0} & \mu_{1} & \cdots & \mu_{n} \\
\mu_{-1} & \mu_{0} & \cdots & \mu_{n-1} \\
\vdots & \vdots & & \vdots \\
\mu_{-n+1} & \mu_{-n+2} & \cdots & \mu_{1} \\
z^{n} & z^{n-1} & \cdots & 1
\end{array}\right|, \\
Q_{2 n+1}(z)=\frac{1}{T_{n}^{(0)}}\left|\begin{array}{cccc}
\mu_{0} & \mu_{-1} & \cdots & \mu_{-n} \\
\mu_{1} & \mu_{0} & \cdots & \mu_{-n+1} \\
\vdots & \vdots & & \vdots \\
\mu_{n-1} & \mu_{n-2} & \cdots & \mu_{1} \\
1 & z & \cdots & z^{n}
\end{array}\right| .
\end{array}
$$

(B) Conversely, let $\left(L_{0}, L_{\infty}\right)$ be a pair of formal power series (2.5) such that $\left\{\mu_{k}\right\}_{-\infty}^{\infty}$ satisfies (2.8). Let $\left\{\delta_{n}\right\}$ be defined by

$$
\delta_{0}=\mu_{0}, \quad \delta_{n}=(-1)^{n} \frac{T_{n}^{(-1)}}{T_{n}^{(0)}}, \quad n=1,2,3, \ldots .
$$


Then $\left|\delta_{n}\right|<1$ for $n=1,2,3, \ldots$, and hence (1.3a) is a PPC-fraction whose coefficients $\delta_{n}$ satisfy equations (2.9), and (1.3) corresponds to $\left(L_{0}, L_{\infty}\right)$.

\section{Proof.}

(A) Combining equation (2.3c) with Theorem 2.1 yields, for $n=$ $1,2,3, \ldots$,

$$
Q_{2 n}(z) L_{0}-P_{2 n}(z)=-2 \delta_{0} \overline{\delta_{n+1}} \prod_{j=1}^{n}\left(1-\left|\delta_{j}\right|^{2}\right) z^{n+1}+O\left(z^{n+2}\right)
$$

and

$$
Q_{2 n}(z) L_{\infty}-P_{2 n}(z)=-2 \delta_{0} \prod_{j=1}^{n}\left(1-\left|\delta_{j}\right|^{2}\right)+O\left(\frac{1}{z}\right) .
$$

By equating coefficients of like powers of $z$ on both sides of equations $(2.12 \mathrm{a})$ and $(2.12 \mathrm{~b})$, we arrive at the system of linear equations

$$
\begin{array}{rllll}
\mu_{0}+ & \mu_{-1} q_{2 n, 1} & +\cdots+ & \mu_{-n} q_{2 n, n} & =\delta_{0} \prod_{j=1}^{n}\left(1-\left|\delta_{j}\right|^{2}\right) \\
\mu_{1}+\mu_{0} q_{2 n, 1} & +\cdots+ & \mu_{-n+1} q_{2 n, n} & =0 \\
& \vdots & \vdots & \vdots \\
\mu_{n}+\mu_{n-1} q_{2 n, 1} & +\cdots+\mu_{0} q_{2 n, n} & =0 .
\end{array}
$$

Since a unique solution to the system (2.13) is ensured by Theorem 2.1, the Toeplitz determinants of the system satisfy $T_{n+1}^{(0)} \neq 0$. Cramer's rule [27] implies

$$
T_{n+1}^{(0)}=\delta_{0} \prod_{j=1}^{n}\left(1-\left|\delta_{j}\right|^{2}\right) T_{n}^{(0)}, \quad n=1,2,3, \ldots
$$

Since $T_{1}^{(0)}=\mu_{0}=\delta_{0}>0$, it follows by induction that $T_{n}^{(0)}>0$ for $n=1,2,3, \ldots$ The expression for $\delta_{n}$ given in equation (2.9) can be obtained by solving the last $n$ equations in the system (2.13) for $q_{2 n, n}$ since $\overline{\delta_{n}}=q_{2 n, n}, \mu_{n}=\overline{\mu_{-n}}$, and hence $T_{n}^{(1)}=\overline{T_{n}^{(-1)}}$. The expression for $\left(1-\left|\delta_{n}\right|^{2}\right)$ in equation (2.9) is a consequence of the Jacobi identities [23]

$$
\left(T_{n}^{(0)}\right)^{2}=T_{n+1}^{(0)} T_{n-1}^{(0)}+T_{n}^{(1)} T_{n}^{(-1)}, \quad n=1,2,3, \ldots
$$


and the expressions for $\delta_{n}$ and $\overline{\delta_{n}}$ in equation (2.9). The formulas given in equation (2.10) can be derived from the last $n$ equations in the system (2.13).

(B) Since $\left\{\mu_{k}\right\}$ satisfies relationship (2.8), we have $T_{n}^{(1)}=\overline{T_{n}^{(-1)}}$, and hence by (2.15),

$$
1-\left|\delta_{n}\right|^{2}=\frac{T_{n+1}^{(0)} T_{n-1}^{(0)}}{\left(T_{n}^{(0)}\right)^{2}}>0, \quad n=1,2,3, \ldots .
$$

Therefore, (1.3a) is a PPC-fraction, and it corresponds to a pair $\left(\widehat{L}_{0}, \widehat{L}_{\infty}\right)$ of the formal power series

$$
\widehat{L}_{0}=\widehat{\mu}_{0}+2 \sum_{k=1}^{\infty} \widehat{\mu}_{k} z^{k}, \quad \widehat{L}_{\infty}=-\widehat{\mu}_{0}-2 \sum_{k=1}^{\infty} \widehat{\mu}_{-k} z^{-k} .
$$

If $\widehat{T}_{k}^{(m)}$ denotes the Toeplitz determinant associated with $\left\{\widehat{\mu}_{k}\right\}_{-\infty}^{\infty}$, then one can show that $\widehat{T}_{K}^{(m)}=T_{k}^{(m)}$, for $m=0, \pm 1, \pm 2, \ldots$ and $k=1,2,3, \ldots$ by a standard argument; see, e.g., [42, Theorem 7.2]. Therefore, (3.1a) is a PPC-fraction corresponding to $\left(L_{0}, L_{\infty}\right)$.

An important characteristic of continued fractions is that the approximants can be generated by the composition of a sequence of linear fractional transformations. This property has been exploited in the development of continued fraction convergence theory $[\mathbf{4 2}, \mathbf{4 9}, \mathbf{5 0}]$.

By use of conformal mapping one can verify the convergence of the approximant sequences $\left\{f_{n}(z)\right\}$ and $\left\{g_{n}(z)\right\}$ and also estimate the truncation error.

The linear fractional transformations associated with a PPC-fraction (1.3) follow from equations (1.7)-(1.9) and are, for $n=1,2,3, \ldots$,

$$
\begin{aligned}
& s_{0}(z, \omega):=\delta_{0}+\omega, \quad s_{2 n}(z, \omega):=\frac{1}{\overline{\delta_{n}} z+\omega}, \\
& s_{1}(z, \omega):=\frac{-2 \delta_{0}}{1+\omega}, s_{2 n+1}(z, \omega):=\frac{\left(1-\left|\delta_{n}\right|^{2}\right) z}{\delta_{n}+\omega}, \\
& S_{0}(z, \omega):=s_{0}(z, \omega), \quad S_{n}(z, \omega):=S_{n-1}\left(z, s_{n}(z, \omega)\right),
\end{aligned}
$$




$$
\begin{gathered}
r_{0}(z, \omega):=s_{0}\left(z, s_{1}\left(z, \omega^{-1}\right)\right)=\delta_{0} \frac{1-\omega}{1+\omega}, \\
r_{n}(z, \omega):=\frac{1}{s_{2 n}\left(z, s_{2 n+1}\left(z, \omega^{-1}\right)\right)}=z \frac{\overline{\delta_{n}}+\omega}{1+\delta_{n} \omega}, \\
R_{0}(z, \omega):=r_{0}(z, \omega), \quad R_{n}(z, \omega):=R_{n-1}\left(z, r_{n}(z, \omega)\right) .
\end{gathered}
$$

It follows that, for $n=1,2,3, \ldots$,

$$
S_{n}(z, \omega)=\frac{P_{n}(z)+\omega P_{n-1}(z)}{Q_{n}(z)+\omega Q_{n-1}(z)},
$$

$$
R_{n}(z, \omega)=S_{2 n+1}\left(z, \omega^{-1}\right)=\frac{P_{2 n+1}(z) \omega+P_{2 n}(z)}{Q_{2 n+1}(z) \omega+Q_{2 n}(z)} .
$$

Hence, for $n=1,2,3, \ldots$,

$$
f_{n}(z):=\frac{P_{2 n}(z)}{Q_{2 n}(z)}=R_{n}(z, 0), \quad g_{n}(z):=\frac{P_{2 n+1}(z)}{Q_{2 n+1}(z)}=R_{n}(z, \infty) .
$$

Theorem 2.3 ([32, 44], Convergence). Let (1.3) be a PPC-fraction with corresponding pair $\left(L_{0}, L_{\infty}\right)$ of formal power series (2.5) and $(2 n)$ th approximant $f_{n}(z)=P_{2 n}(z) / Q_{2 n}(z)$. Then $\left\{f_{n}(z)\right\}_{0}^{\infty}$ converges uniformly on compact subsets of the unit disk $|z|<1$ to a normalized Carathéodory function $f(z)$ satisfying, for $n=1,2,3, \ldots$,

$$
\begin{gathered}
f(0)=\delta_{0}=\mu_{0}>0, \quad \operatorname{Re} f(z)>0, \quad \text { for }|z|<1, \\
f(z)=\mu_{0}+2 \sum_{k=1}^{\infty} \mu_{k} z^{k}, \quad \text { for }|z|<1, \\
\left|f(z)-\delta_{0} \frac{1+\rho^{2}}{1-\rho^{2}}\right| \leq \frac{2 \delta_{0} \rho}{1-\rho^{2}}, \quad \text { for }|z|<\rho<1, \\
\left|f(z)-f_{n}(z)\right| \leq \frac{4 \delta_{0}|z|^{n+1}}{1-|z|^{2}}, \quad \text { for }|z|<1, \\
\left|f(z)-f_{n}(z)\right| \leq \frac{4 \delta_{0} \prod_{j=1}^{n}\left(1-\left|\delta_{j}\right|^{2}\right)|z|^{n+1}}{\left|Q_{2 n}(z)\right|^{2}-\left|z Q_{2 n+1}(z)\right|^{2}}, \quad \text { for }|z|<1 .
\end{gathered}
$$


The inequalities (2.23) and (2.24) provide a priori and a posteriori truncation error bounds, respectively. Our proof of Theorem 2.3 makes use of four lemmas.

Lemma 2.4 (Conformal mapping). Let (1.3) be a PPC-fraction with associated linear fractional transformations (2.16). For $R:=|z|<1$, let $U_{R}$ be the open disk in $\mathbb{C}$ defined by $U_{R}:=[u:|u|<R]$. Let

$$
\Gamma_{n}:=\frac{\overline{\delta_{n}}\left(1-R^{2}\right) z}{1-R^{2}\left|\delta_{n}\right|^{2}}, \quad \rho_{n}:=\frac{\left(1-\left|\delta_{n}\right|^{2}\right) R^{2}}{1-R^{2}\left|\delta_{n}\right|^{2}}, \quad n=1,2,3, \ldots
$$

Then

$$
r_{0}\left(z, U_{R}\right)=\left[\xi \in \mathbb{C}:\left|\xi-\delta_{0} \frac{1+R^{2}}{1-R^{2}}\right|<\frac{2 \delta_{0} R}{1-R^{2}}\right]
$$

and

$$
r_{n}\left(z, U_{R}\right)=\left[\xi \in \mathbb{C}:\left|\xi-\Gamma_{n}\right|<\rho_{n}\right] \subseteq U_{R}, \quad n=1,2,3, \ldots
$$

Proof. It is readily shown that, if $\xi=r_{0}(z, \omega)$, then $\omega=\left(\delta_{0}-\right.$ $\xi) /\left(\delta_{0}+\xi\right)$, from which one can verify equation (2.26). Similarly, if $\xi=r_{n}(z, \omega)$, then $\omega=\left(\xi-\overline{\delta_{n}} z\right) /\left(z-\delta_{n} \xi\right)$, from which one can obtain the equality relation in (2.27). To prove the inclusion relation in (2.27), it suffices to show that

$$
\left|\Gamma_{n}\right|+\rho_{n} \leq R .
$$

Substituting the expressions for $\Gamma_{n}$ and $\rho_{n}$ in the inequality (2.28) and multiplying both sides by $\left(1-R^{2}\left|\delta_{n}\right|^{2}\right)$ yields the equivalent inequality

$$
R(1-R)\left(1-R\left|\delta_{n}\right|\right) \geq 0,
$$

which is clearly valid.

It follows from Lemma 2.4 and equation (2.16f) that, for $|z|<1$ and $n=1,2,3, \ldots$,

$$
R_{n}\left(z, U_{R}\right) \subseteq R_{n-1}\left(z, U_{R}\right) \subseteq \cdots \subseteq R_{0}\left(z, U_{R}\right)=r_{0}\left(z, U_{R}\right) .
$$

Therefore, $\left\{R_{n}\left(z, U_{R}\right)\right\}$ is a nested sequence of non-empty circular disks. Since

$$
f_{n+m}(z)=R_{n+m}(z, 0) \in R_{n}\left(z, U_{R}\right), \quad n, m=0,1,2, \ldots,
$$


we have the following lemma.

Lemma 2.5. If $|z|<1$, then

$$
\left|f_{n+m}(z)-f_{n}(z)\right| \leq 2 \rho\left(R_{n}\left(z, U_{R}\right)\right), \quad n, m=0,1,2, \ldots,
$$

where $\rho(D)$ denotes the radius of a disk $D$.

It follows that the sequence $\left\{f_{n}(z)\right\}$ converges to a finite value whenever we have $\lim _{n \rightarrow \infty} \rho\left(R_{n}\left(z, U_{R}\right)\right)=0$.

Lemma 2.6. For $R=|z|<1$ and $n=1,2,3, \ldots$,

$$
\rho\left(R_{n}\left(z, U_{R}\right)\right)=\frac{2 \delta_{0} \prod_{j=1}^{n}\left(1-\left|\delta_{j}\right|^{2}\right)|z|^{n+1}}{\left|Q_{2 n}(z)\right|^{2}-\left|z Q_{2 n+1}(z)\right|^{2}} .
$$

Proof. Let $\omega_{n} \in U_{R}$ be chosen so that $R_{n}\left(z, \omega_{n}\right)$ is the center of the disk $R_{n}\left(z, U_{R}\right)$. By equation (2.18),

$$
R_{n}\left(z,-u_{n}\right)=\infty \quad \text { if } u_{n}:=\frac{Q_{2 n}(z)}{Q_{2 n+1}(z)} .
$$

Since $R_{n}\left(z, \omega_{n}\right)$ and $R_{n}\left(z,-u_{n}\right)$ are inverses with respect to the boundary of $R_{n}\left(z, U_{R}\right)$, and since inverses are preserved under linear fractional transformations, it follows that $\omega_{n}$ and $-u_{n}$ are inverses with respect to the circle $|\omega|=R=|z|$ in the $\omega$-plane. Hence, the ray extending from $\omega=0$ to $\omega=\omega_{n}$ passes through $\omega=-u_{n}$ and

$$
\tau:=\operatorname{Arg}\left(\omega_{n}\right)=\operatorname{Arg}\left(-u_{n}\right) \text { and }\left|\omega_{n}\right| \cdot\left|u_{n}\right|=R^{2}=|z|^{2}<1
$$

Therefore, $\nu_{n}:=|z| e^{i \tau_{n}}$ is the point of intersection of the circle $|\omega|=R$ and the line segment $\left[\omega_{n},-u_{n}\right]$. An application of equation (2.18) and the determinant formulas (2.3) yields

$$
\begin{aligned}
\rho\left(R_{n}\left(z, U_{R}\right)\right) & =\left|R_{n}\left(z, \omega_{n}\right)-R_{n}\left(z, \nu_{n}\right)\right| \\
& =\frac{2 \delta_{0} \prod_{j=1}^{n}\left(1-\left|\delta_{j}\right|^{2}\right)|z|^{n}\left|\omega_{n}-\nu_{n}\right|}{\left|Q_{2 n}(z)\right|^{2}\left|\omega_{n}+u_{n}\right| \cdot\left|u_{n}+\nu_{n}\right|} .
\end{aligned}
$$

By equations (2.33) and (2.34), we obtain

$$
\left|\omega_{n}-\nu_{n}\right|=\left(\left|Q_{2 n}(z)-\right| z Q_{2 n+1}(z) \mid\right)|z|=\left|u_{n}+\nu_{n}\right| \cdot|z|
$$


and

$$
\left|\omega_{n}+u_{n}\right|=\frac{\left|Q_{2 n}(z)\right|^{2}-\left|z Q_{2 n+1}(z)\right|^{2}}{\left|Q_{2 n}(z) Q_{2 n+1}(z)\right|},
$$

from which equation (2.32) is an immediate consequence.

It is convenient to introduce $\varrho_{0}:=1 / \mu_{0}$ and for $n=1,2,3, \ldots$,

$$
\varrho_{n}:=\frac{T_{n}^{(0)}}{T_{n+1}^{(0)}}=\frac{\varrho_{n-1}}{1-\left|\delta_{n}\right|^{2}}=\frac{\varrho_{0}}{\prod_{j=1}^{n}\left(1-\left|\delta_{j}\right|^{2}\right)},
$$

which follows from equation (2.9).

Lemma 2.7 (Christoffel-Darboux formulas). For $x, y \in \mathbb{C}, x \bar{y} \neq 1$ and $n=0,1,2, \ldots$,

$$
\begin{aligned}
\sum_{j=0}^{n} \varrho_{j} Q_{2 j-1}(x) \overline{Q_{2 j-1}(y)} & \\
= & \frac{\varrho_{n}\left(Q_{2 n}(x) \overline{Q_{2 n}(y)}-x \bar{y} Q_{2 n+1}(x) \overline{Q_{2 n+1}(y)}\right)}{1-x \bar{y}}
\end{aligned}
$$

Proof. For $n=1,2,3, \ldots$, the difference equations (1.10) imply

$$
\begin{gathered}
Q_{2 n+1}(z)=z Q_{2 n-1}(z)+\delta_{n} Q_{2 n-2}(z), \\
Q_{2 n}(z)=\bar{\delta}_{n} z Q_{2 n-1}(z)+Q_{2 n-2}(z),
\end{gathered}
$$

and hence,

$$
z Q_{2 n-1}(z)=\frac{Q_{2 n+1}(z)-\delta_{n} Q_{2 n}(z)}{1-\left|\delta_{n}\right|^{2}}
$$

and

$$
Q_{2 n-2}(z)=\frac{Q_{2 n}(z)-\bar{\delta}_{n} Q_{2 n+1}(z)}{1-\left|\delta_{n}\right|^{2}}
$$


From equations (2.37) and (2.38), it follows that

$$
\begin{aligned}
& \frac{\varrho_{j}\left(Q_{2 j}(x) \overline{Q_{2 j}(y)}-x \bar{y} Q_{2 j+1}(x) \overline{Q_{2 j+1}(y)}\right)}{1-x \bar{y}} \\
& =\frac{\varrho_{j}\left(Q_{2 j-2}(x) \overline{Q_{2 j-2}(y)}-x \bar{y} Q_{2 j-1}(x) \overline{Q_{2 j-1}(y)}\right)}{1-x \bar{y}} \\
& \quad+\varrho_{j} Q_{2 j+1}(x) \overline{Q_{2 j+1}(y)} .
\end{aligned}
$$

Summing both sides of equation (2.39) yields the Christoffel-Darboux formulas (2.36).

Proof of Theorem 2.3. In equation (2.36), we set $x=y=z$ and obtain

$$
\varrho_{0} \leq \sum_{j=0}^{n} \varrho_{j}\left|Q_{2 j+1}(z)\right|^{2}=\frac{\varrho_{n}\left(\left|Q_{2 n}(z)\right|^{2}-\left|z Q_{2 n+1}(z)\right|^{2}\right)}{1-|z|^{2}}
$$

Therefore, by equation (2.35),

$$
\left|Q_{2 n}(z)\right|^{2}-\left|z Q_{2 n+1}(z)\right|^{2} \geq\left(1-|z|^{2}\right) \prod_{j=1}^{n}\left(1-\left|\delta_{j}\right|^{2}\right) .
$$

Combining equation (2.32) and inequality (2.41) yields, for $|z|=R<1$,

$$
\operatorname{rad} \partial R_{n}\left(z, U_{R}\right) \leq \frac{2 \delta_{0} \prod_{j=1}^{n}\left(1-\left|\delta_{j}\right|^{2}\right)|z|^{n+1}}{\left|Q_{2 n}(z)\right|^{2}-\left|z Q_{2 n+1}(z)\right|^{2}} \leq \frac{2 \delta_{0}|z|^{n+1}}{1-|z|^{2}}
$$

It follows from inequality (2.42) and Lemma 2.5 that $\left\{f_{n}(z)\right\}_{0}^{\infty}$ converges uniformly on compact subsets of $|z|<1$ to a function $f(z)$ holomorphic in $|z|<1$. The mapping properties (2.29) imply that Re $f(z)>0$ for $|z|<1$, and hence, $f(z)$ is a normalized Carathéodory function. The truncation error estimates (2.23) and (2.24) follow from inequality (2.42). By a convergence theorem for continued fractions given in [42, Theorem 5.13], assertion (2.21) holds since the pair $\left(L_{0}, L_{\infty}\right)$ of power series $(2.5)$ corresponds to the PPC-fraction (1.3) and the sequence $\left\{f_{n}(z)\right\}_{0}^{\infty}$ is uniformly bounded on compact subsets of $|z|<1$. 
We note that the convergence theorem [42, Theorem 5.13] makes essential use of the Stieltjes-Vitali theorem (see, e.g., [25, Theorem 15.3.2], [71], [74, Theorem 20.15]).

From equation (2.2) and Theorem 2.3 it follows that the sequence $\left\{g_{n}(z)\right\}$ of odd order approximants of a PPC-fraction (1.1) converges to a function $g(z)$ holomorphic in $|z|>1$, satisfying

$$
g(0)=-\mu_{0}=-\delta_{0}<0, \quad \operatorname{Re}(g(z))<0 \quad \text { for }|z|>1,
$$

and

$$
g(z)=-\mu_{0}-2 \sum_{k=1}^{\infty} \mu_{-k} z^{-k}, \quad|z|>1
$$

\section{Trigonometric moment problem.}

Theorem 3.1 ([32], [34], Trigonometric moment problem). Let $\left\{\mu_{n}\right\}_{-\infty}^{\infty}$ be a doubly infinite sequence in $\mathbb{C}$ with associated Toeplitz determinants $T_{k}^{(m)}$ given by equation (1.17). Then the following three statements are equivalent.

(A) There exists a distribution function $\psi \in \Psi_{\infty}(-\pi, \pi)$ such that its moments $\mu_{n}$ satisfy

$$
\mu_{n}=\frac{1}{2 \pi} \int_{-\pi}^{\pi} e^{-i n \theta} d \psi(\theta), \quad n=0, \pm 1, \pm 2, \ldots
$$

$$
\mu_{n}=\overline{\mu_{-n}} \quad \text { and } \quad T_{n}^{(0)}>0, \quad n=1,2,3, \ldots .
$$

(C) There exists a PPC-fraction (1.3) corresponding to the pair $\left(L_{0}, L_{\infty}\right)$ of power series $(2.5)$.

Proof. We begin by showing that (A) implies (B). If there exists a $\psi \in \Psi_{\infty}(-\pi, \pi)$ such that equation $(3.1)$ holds, then clearly $\mu_{n}=\overline{\mu_{-n}}$, $n=0,1,2, \ldots$. If

$$
P(z)=\sum_{k=-n}^{n} a_{k} z^{k}, \quad a_{k} \in \mathbb{C}
$$


then

$$
\frac{1}{2 \pi} \int_{-\pi}^{\pi}\left|P\left(e^{i \theta}\right)\right|^{2} d \psi(\theta)=\sum_{j, k=-n}^{n} a_{j} \overline{a_{k}} \mu_{k-j} \geq 0,
$$

and the left hand side of equation (3.4) equals zero if and only if $P(z) \equiv 0$, since $\psi(\theta)$ has infinitely many points of increase. Therefore, the right hand side of equation (3.3) is a positive definite Toeplitz form. By a well-known property of Toeplitz forms, $T_{n}^{(0)}>0, n=1,2,3, \ldots$, [21, pages 16-19].

The equivalence of (B) and (C) is implied by Theorem $2.2(\mathrm{~B})$.

It remains to show that (C) implies (A). By Theorem 2.3, the sequence of $(2 n)$ approximants $\left\{f_{n}(z)\right\}$ of the PPC-fraction (1.3) converges to a normalized Carathéodory function $f(z)$. It follows from the Herglotz-Riesz representation theorem [1, page 91] that there exists a $\psi \in \Psi(-\pi, \pi)$, such that

$$
f(z)=\frac{1}{2 \pi} \int_{-\pi}^{\pi} \frac{e^{i \theta}+z}{e^{i \theta}-z} d \psi(\theta), \quad|z|<1 .
$$

Expanding the integrand of equation (3.5) in increasing powers of $z e^{-i \theta}$ and integrating term-by-term yields

$$
f(z)=\mu_{0}+2 \sum_{k=1}^{\infty} \mu_{k} z^{k}, \quad \text { for }|z|<1,
$$

where the $\mu_{k}$ are given by equation (3.1). It remains to show that $\psi(\theta)$ has infinitely many points of increase. It $\psi(\theta)$ has only a finite number of points of increase, then there exists a Laurent polynomial (3.3), not identically zero, such that the Toeplitz form (3.4) is zero. This implies that $T_{n}^{(0)}=0$ for some $n \in[1,2,3, \ldots]$, and hence, the inequality in (3.2) does not hold. This leads to a contradiction since (B) and (C) are equivalent.

The $M$-definite trigonometric moment problem in which $\psi(\theta)$ has only a finite number $M$ of points of increase is treated in Section 5 using $M$-terminating PPC-fractions.

4. Szegö polynomials. Since the Szegö polynomials $\rho_{n}(z)$ and reciprocal polynomials $\rho_{n}^{*}(z)$ can be expressed as denominators of a 
PPC-fraction, one can easily derive many properties of $\rho_{n}(z)$ and $\rho_{n}^{*}(z)$ from corresponding properties of PPC-fractions.

Theorem 4.1. Let $\psi \in \Psi_{\infty}(-\pi, \pi)$ be given, let $\langle\cdot, \cdot\rangle_{\psi}$ be the associated inner product (1.21), and let $\mathrm{PPC}\left\{\delta_{n}\right\}$ be the PPC-fraction whose existence is insured by Theorem 3.1. Let $\left\{\rho_{n}(z)\right\}_{0}^{\infty}$ and $\left\{\rho_{n}^{*}(z)\right\}_{0}^{\infty}$ be defined by

$$
\rho_{n}(z):=Q_{2 n+1}(z), \quad \rho_{n}^{*}(z)=Q_{2 n}(z), \quad n=0,1,2, \ldots
$$

Then, for $n \geq 0, \rho_{n}(z)$ is a monic polynomial of degree $n$ and

$$
\begin{gathered}
\left\langle\rho_{n}(z), z^{m}\right\rangle_{\psi}= \begin{cases}0 & m=0,1, \ldots, n-1 \\
T_{n+1}^{(0)} / T_{n}^{(0)} & m=n,\end{cases} \\
\left\langle\rho_{n}^{*}(z), z^{m}\right\rangle_{\psi}= \begin{cases}T_{n+1}^{(0)} / T_{n}^{(0)} & m=0, \\
0 & m=1,2, \ldots, n,\end{cases} \\
\rho_{n}(z)=z \rho_{n-1}(z)+\delta_{n} \rho_{n-1}^{*}(z), \\
\rho_{n}^{*}(z)=\overline{\delta_{n}} z \rho_{n-1}(z)+\rho_{n-1}^{*}(z), \\
\rho_{n}^{*}(z)=z^{n} \overline{\rho_{n}(1 / \bar{z})} \quad \text { and } \quad \rho_{n}(z)=z^{n} \overline{\rho_{n}^{*}(1 / \bar{z})} \\
\Longrightarrow|z|<1 .
\end{gathered}
$$

Proof. Let $\left\{\mu_{n}\right\}_{0}^{\infty}$ be the moment sequence (3.1) associated with $\psi$. By equation (4.1) and Theorem $2.2(\mathrm{~A}), \rho_{n}(z)$ and $\rho_{n}^{*}(z)$ can be expressed in terms of Toeplitz determinants $T_{k}^{(m)}$ as in equation (2.10). It follows from equation (2.10) that $\rho_{n}(z)$ is a monic polynomial of degree $n$ and $\rho_{n}^{*}(z)$ is a polynomial of degree at most $n$. The orthogonality properties (4.2) and (4.3) follow from equations (2.10) and (3.1). Recurrence relations (4.4) are readily derived from equation (4.1), and the difference equations (1.10). The reciprocity relation (4.5) follows from equations (2.1) and (4.1). It remains only to verify (4.6). From 
the mapping properties (2.26), (2.27) and (2.29), we have

$$
\left|\frac{P_{2 n+1}(z)}{Q_{2 n+1}(z)}-\delta_{0} \frac{p^{2}+1}{p^{2}-1}\right| \leq \frac{2 \delta_{0} p}{p^{2}-1}, \quad \text { for }|z| \geq p>1 .
$$

Thus, all zeros of $\rho_{n}(z)=Q_{2 n+1}(z)$ lie inside $|z| \leq 1$. Let

$$
\rho_{n}(z)=\prod_{j=1}^{n}\left(z-z_{j}\right) \quad \text { and } \quad \rho_{n}^{*}(z)=\prod_{j=1}^{n}\left(1-\overline{z_{j}} z\right),
$$

where $z_{1}, z_{2}, \ldots, z_{n}$ are the zeros of $\rho_{n}(z)$. Assume that one of the zeros, say $z_{k}=e^{i \theta_{k}}$, lies on $|z|=1$. From equation (4.8), we obtain $\rho_{n}\left(z_{k}\right)=$ $0=\rho_{n}^{*}\left(z_{k}\right)$, which leads to a contradiction of equation (2.3a).

It follows from (4.6) that all $n$ zeros of $\rho_{n}(z)$ lie in the open disk $|z|<1$. From equation (4.5), if $z_{k} \neq 0$ is a zero of $\rho_{n}(z)$, then $1 / \bar{z}_{k}$ is a zero of $\rho_{n}^{*}(z)$. Hence, all zeros of $\rho_{n}^{*}(z)$ lie in $|z|>1$.

Levinson's algorithm [47] is an efficient procedure for the computation of the coefficients of individual powers of $z$ in Szegö polynomials and the $\delta_{n}$ coefficients of associated PPC-fractions. The algorithm is of great value for frequency analysis computation. For the Szegö polynomials $\rho_{n}(z)$ associated with a distribution function $\psi \in \Psi_{\infty}(-\pi, \pi)$, we write

$$
\rho_{n}(z)=\sum_{j=0}^{n} q_{j}^{(n)} z^{j}, \quad \rho_{n}^{*}(z)=\sum_{j=0}^{n} \overline{q_{j}^{(n)}} z^{n-j}, \quad q_{n}^{(n)}=1 .
$$

Then by equations (4.2) and (4.4a), for $n=1,2,3, \ldots$,

$$
0=\left\langle\rho_{n}(z), 1\right\rangle_{\psi}=\left\langle z \rho_{n-1}(z), 1\right\rangle_{\psi}+\delta_{n}\left\langle\rho_{n-1}^{*}(z), 1\right\rangle_{\psi},
$$

and hence,

$$
\delta_{n}=-\frac{\sum_{j=0}^{n-1} q_{j}^{(n-1)} \mu_{-j-1}}{\sum_{j=0}^{n-1} \overline{q_{j}^{(n-1)}} \mu_{j+1-n}}, \quad n=1,2,3, \ldots .
$$

Since $\rho_{0}(z)=\rho_{0}^{*}(z)=1$, equation (4.10) yields

$$
\delta_{1}=-\frac{\mu_{-1}}{\mu_{0}} .
$$


From equation (4.4),

$$
q_{1}^{(1)}=\overline{q_{1}^{(1)}}=1, \quad q_{0}^{(1)}=-\frac{\mu_{-1}}{\mu_{0}}, \quad \overline{q_{0}^{(1)}}=-\frac{\mu_{1}}{\mu_{0}},
$$

and hence, by equation (4.10),

$$
\delta_{2}=\frac{\mu_{-1}^{2}-\mu_{0} \mu_{-2}}{\mu_{0}^{2}-\mu_{1} \mu_{-1}} .
$$

Continuing in this manner, one can calculate successively the coefficients $\delta_{n}, q_{j}^{(n)}, n=1,2,3, \ldots$

Theorem 4.2. Let $P(z)$ be a polynomial in $z$ of the form

$$
P(z)=z^{n}+a_{1} z^{n-1}+a_{2} z^{n-2}+\cdots+a_{n}, \quad a_{j} \in \mathbb{C}, n \geq 1,
$$

and let $\psi \in \Psi_{\infty}(-\pi, \pi)$. Then,

$$
\min _{a_{j} \in \mathbb{C}} \frac{1}{2 \pi} \int_{-\pi}^{\pi}\left|P\left(e^{i \theta}\right)\right|^{2} d \psi(\theta)=\frac{1}{2 \pi} \int_{-\pi}^{\pi}\left|\rho_{n}\left(e^{i \theta}\right)\right|^{2} d \psi(\theta)=\left\langle\rho_{n}, \rho_{n}\right\rangle_{\psi},
$$

where $\rho_{n}(z)$ is the nth degree, monic Szegö polynomial with respect to $\psi$.

Proof. See, e.g., [72, Theorem 11.1.2].

5. M-terminating PPC-fractions. The results of Sections 2, 3 and 4 have immediate counterparts for M-terminating PPC-fractions (1.14). These are summarized in the present section, with proofs being given only when they differ significantly from the analogous results for PPC-fractions.

The $n$th numerator $P_{n}(z)$ and denominator $Q_{n}(z)$ are polynomials defined by the difference equations (1.10) for $n=0,1,2, \ldots, M$. Since $\left|\delta_{M}\right|=1$, we have

$$
\begin{array}{cl}
P_{2 M+1}(z)=\delta_{M} P_{2 M}(z), & Q_{2 M+1}(z)=\delta_{M} Q_{2 M}(z), \\
P_{2 M}(z)=-\overline{\delta_{M}} z^{M} \overline{P_{2 M}(1 / \bar{z}),} & Q_{2 M}(z)=\overline{\delta_{M}} z^{M} \overline{Q_{2 M}(1 / \bar{z})}
\end{array}
$$

and

$$
f(z):=\frac{P_{2 M}(z)}{Q_{2 M}(z)}=\frac{P_{2 M+1}(z)}{Q_{2 M+1}(z)}=-\overline{f(1 / \bar{z})}
$$


Theorem 5.1. Let $f(z)$ be a rational function represented by an Mterminating PPC-fraction (1.14). Then:

(A) $f(z)$ is holomorphic for $|z|<1$ and for $|z|>1$ and satisfies

(5.4) $f(0)>0, \quad \operatorname{Re} f(z)>0$ for $|z|<1, \quad \operatorname{Re} f(z)<0$ for $|z|>1$.

(B) $f(z)$ has power series representations of the form

$$
f(z)= \begin{cases}\mu_{0}+2 \sum_{k=1}^{\infty} \mu_{k} z^{k} & |z|<1 \\ -\mu_{0}-2 \sum_{k=1}^{\infty} \mu_{-k} z^{-k} & |z|>1\end{cases}
$$

where the coefficients $\mu_{k}$ satisfy

$$
\begin{gathered}
\mu_{n}=\overline{\mu_{-n}} \quad \text { and } T_{n}^{(0)}>0 \\
\text { for } n=0,1,2, \ldots M \quad \text { and } T_{M+1}^{(0)}=0 .
\end{gathered}
$$

(C) The coefficients $\delta_{n}$ in the M-terminating PPC-fraction (1.14) satisfy

$$
\delta_{0}=\mu_{0}>0
$$

and, for $n=1,2, \ldots, M$,

$$
\delta_{n}=(-1)^{n} \frac{T_{n}^{(-1)}}{T_{n}^{(0)}} \quad \text { and } \quad 1-\left|\delta_{n}\right|^{2}=\frac{T_{n+1}^{(0)} T_{n-1}^{(0)}}{\left(T_{n}^{(0)}\right)^{2}} .
$$

(D) The nth denominators $Q_{n}(z)$ of the M-terminating PPC fraction (1.14) are represented by the determinant formulas (2.10) for $n=1,2, \ldots, M$.

(E) The $\mathrm{M}$ zeros of $Q_{2 M+1}(z)=\delta_{M} Q_{2 M}(z)$ lie on the unit circle $|z|=1$. It will be shown (Theorem 5.4) that they are distinct and non-real zeros occurring in conjugate pairs.

Theorem 5.2. Let $\left\{\mu_{n}\right\}_{-\infty}^{\infty}$ be a doubly infinite sequence in $\mathbb{C}$ satisfying equation (5.6). Let $\left\{\delta_{n}\right\}_{0}^{\infty}$ be defined by

$$
\delta_{0}:=\mu_{0} \quad \text { and } \quad \delta_{n}:=(-1)^{n} \frac{T_{n}^{(-1)}}{T_{n}^{(0)}}, n=1,2, \ldots M .
$$

Then $\left\{\delta_{n}\right\}_{0}^{\infty}$ satisfies equation (1.14b); hence, equation (1.14a) is an $\mathrm{M}$-terminating $\mathrm{PPC}$-fraction representing a function $f(z)$ with power series expansions (5.5). 
Theorem 5.3. Let $\left\{\mu_{n}\right\}_{-\infty}^{\infty}$ be an infinite double sequence in $\mathbb{C}$ with Toeplitz determinants $T_{k}^{(m)}$ given by equation (1.17). Then the following three statements are equivalent:

(A) There exists a distribution function $\psi \in \Psi_{M}(-\pi, \pi)$, for some $M \in[1,2,3, \ldots]$, such that

$$
\mu_{n}=\frac{1}{2 \pi} \int_{-\pi}^{\pi} e^{-i n \theta} d \psi(\theta), \quad n=0, \pm 1, \pm 2, \ldots
$$

$\mu_{n}=\overline{\mu_{-n}} \quad$ and $\quad T_{n}^{(0)}>0$ for $n=0,1,2, \ldots, M$ and $T_{M+1}^{(0)}=0$.

(C) There exists an M-terminating PPC-fraction (1.14) representing a normalized Carathéodory function $f(z)$, such that

$$
f(z)=\mu_{0}+2 \sum_{k=1}^{\infty} \mu_{k} z^{k} \quad \text { for }|z|<1
$$

and

$$
f(z)=-\mu_{0}-2 \sum_{k=1}^{\infty} \mu_{-k} z^{-k} \quad \text { for }|z|>1 .
$$

Proof. It follows from a well-known property of Toeplitz forms [21, page 19] that (A) and (B) are equivalent. By Theorem 5.1, (A) and (C) are equivalent.

If $\psi \in \Psi_{M}(-\pi, \pi)$ for $M \in[1,2,3, \ldots]$, then

$$
\langle P(z), Q(z)\rangle_{\psi}:=\frac{1}{2 \pi} \int_{-\pi}^{\pi} P\left(e^{i \theta}\right) \overline{Q\left(e^{i \theta}\right)} d \psi(\theta),
$$

defines an $M$-definite inner product on

$$
\Lambda_{-M-1, M+1}=\left\{\sum_{k=-n}^{n} c_{k} z^{k}: c_{k} \in \mathbb{C}, 0 \leq n \leq M-1\right\} .
$$

Let $Q_{n}(z)$ denote the $n$th denominator of the $M$-terminating PPCfraction (1.14) associated with $\psi$ (Theorem 5.3). Then, for $n=$ 
$0,1,2, \ldots, M$, the $n$th degree monic Szegö polynomial $\rho_{n}(z)$ and $n$th reciprocal polynomial $\rho_{n}^{*}(z)$ are given by:

$$
\rho_{n}(z)=Q_{2 n+1}(z), \quad \rho_{n}^{*}(z)=Q_{2 n}(z), \quad n=0,1,2, \ldots, M .
$$

Basic properties of these polynomials are summarized in the following:

Theorem 5.4. The Szegö polynomials $\rho_{n}(z)$ associated with an Mdefinite inner product (5.12), where $\psi \in \Psi_{M}(-\pi, \pi)$ satisfy the following properties for $n=0,1,2, \ldots, M$ :

$$
\begin{aligned}
\left\langle\rho_{n}(z), z^{k}\right\rangle_{\psi} & = \begin{cases}0 & k=0,1, \ldots, n-1 \\
T_{n+1}^{(0)} / T_{n}^{(0)} & k=n .\end{cases} \\
\left\langle\rho_{n}^{*}(z), z^{k}\right\rangle_{\psi} & = \begin{cases}T_{n+1}^{(0)} / T_{n}^{(0)} & k=0, \\
0 & k=1,2, \ldots, n .\end{cases} \\
\rho_{n}(z) & =z \rho_{n-1}(z)+\delta_{n} \rho_{n-1}^{*}(z), \\
\rho_{n}^{*}(z) & =\overline{\delta_{n}} z \rho_{n-1}(z)+\rho_{n-1}^{*}(z),
\end{aligned}
$$

In addition, $\rho_{M}(z)$ has exactly $M$ zeros, all are distinct and lie on the unit circle. All non-real zeros occur in conjugate pairs.

Proof. Arguments analogous to those used in Section 4 apply, except for the part about the zeros of $\rho_{M}(z)$.

By conditions (5.10) and equation (5.15), $\left\langle\rho_{M}(z), \rho_{M}(z)\right\rangle_{\psi}=0$, and hence, $\rho_{M}\left(e^{i t}\right)=0$ at exactly the $M$ points of increase of $\psi$.

6. Carathéodory functions. PPC-fractions and M-terminating PPC-fractions provide a complete characterization of the normalized Carathéodory functions $f(z) \in \mathcal{C}$. From Theorems 2.3 and 5.1, it is seen that the sequence of $(2 n)$ th approximants of every PPC-fractions 
converges to a function $f(z) \in \mathcal{C}$, and every $M$-terminating PPCfraction represents a function in $\mathcal{C}$. It is now shown that every $f(z) \in \mathcal{C}$ can be represented by one or the other of these two ways.

Theorem 6.1. If $f(z) \in \mathcal{C}$, then either there exists a PPC-fraction with $(2 n)$ th approximants $\left\{f_{n}(z)\right\}$ converging to $f(z)$ for $|z|<1$ or there exists an M-terminating $\mathrm{PPC}$-fraction representing $f(z)$.

Proof. By the Herglotz-Riesz representation theorem [1], there exists $\psi \in \Psi(-\pi, \pi)$ such that

$$
f(z)=\frac{1}{2 \pi} \int_{-\pi}^{\pi} \frac{e^{i \theta}+z}{e^{i \theta}-z} d \psi(\theta)=\mu_{0}+2 \sum_{k=1}^{\infty} \mu_{k} z^{k}, \quad|z|<1,
$$

where $\mu_{k}$ is the $k$ th moment with respect to $\psi$. If $\psi \in \Psi_{\infty}(-\pi, \pi)$, then, by Theorem 3.1, there exists a PPC-fraction with $(2 n)$ th approximant sequence $\left\{f_{n}(z)\right\}$ converging to $f(z)$ uniformly on compact subsets of $|z|<1$. If $\psi \in \Psi_{M}(-\pi, \pi)$ for some positive integer $M$, then by Theorem 5.3, there exists an $M$-terminating PPC-fraction representing $f(z)$.

7. Frequency analysis. Let $B(t)$ be a real valued function of the form (1.24) with normalized frequencies $\omega_{j}$. Let $\psi(\theta)$ be a step function defined on $[-\pi, \pi]$ with a jump $\left|\alpha_{j}\right|^{2}$ at each point $\theta=\omega_{j}$, $j=0, \pm 1, \pm 2, \ldots, \pm I$. Then

$$
\begin{gathered}
\psi \in \Psi_{M}(-\pi, \pi), \quad \text { where } \quad M=2 I+L, \\
L= \begin{cases}0 & \text { if } \alpha=0, \\
1 & \text { if } \alpha=1 .\end{cases}
\end{gathered}
$$

The Herglotz transform [1, page 91]

$$
f(z)=\frac{1}{2 \pi} \int_{-\pi}^{\pi} \frac{e^{i \theta}+z}{e^{i \theta}-z} d \psi(\theta)=\sum_{j=-I}^{I}\left|\alpha_{j}\right|^{2} \frac{e^{i \omega_{j}}+z}{e^{i \omega_{j}}-z}
$$

is a rational, normalized Carathéodory function. If the $k$ th moment with respect to $\psi$ is denoted by $\mu_{k}$, then

$$
f(z)= \begin{cases}\mu_{0}+2 \sum_{k=1}^{\infty} \mu_{k} z^{k} & |z|<1, \\ -\mu_{0}-2 \sum_{k=1}^{\infty} \mu_{-k} z^{-k} & |z|>1 .\end{cases}
$$


By Theorem 6.1, $f(z)$ has an $M$-terminating PPC-fraction representation (1.14)

$$
\begin{aligned}
f(z)= & \delta_{0}-\frac{2 \delta_{0}}{1}+\frac{1}{\overline{\delta_{1}} z}+\frac{\left(1-\left|\delta_{1}\right|^{2}\right) z}{\delta_{1}}+\cdots \\
& +\frac{\left(1-\left|\delta_{M-1}\right|^{2}\right) z}{\delta_{M-1}}+\frac{1}{\overline{\delta_{M}} z} .
\end{aligned}
$$

It follows from equations (7.1) and (7.3) and Theorem 5.4 that the monic $M$ th degree Szegö polynomial with respect to $\psi$ is given by

$$
\rho_{M}(z)=Q_{2 M+1}(z)=(z-1)^{L} \prod_{j=1}^{I}\left(z-e^{i \omega_{j}}\right)\left(z-e^{-i \omega_{j}}\right)
$$

where $Q_{2 M+1}(z)$ is the $(2 M+1)$ th denominator of M-terminating PPCfraction (7.3).

The frequency analysis problem can be solved by determining the critical points $e^{i \omega_{j}}$ on the unit circle; that is, by finding the zeros of $\rho_{M}(z)$.

Let $N$ be an integer greater than $M$, and let $\left\{\chi_{N}(m)\right\}_{-\infty}^{\infty}$ be defined by

$$
\chi_{N}(m)= \begin{cases}B\left(t_{m}\right) & \text { for } m=0,1,2, \ldots, N-1, \\ 0 & \text { for } m<0 \text { or } m \geq N .\end{cases}
$$

An absolutely continuous distribution function $\psi_{N}(\theta) \in \Psi_{\infty}(-\pi, \pi)$ is determined, up to an additive constant, by

$$
\psi_{N}^{\prime}(\theta)=\left|\sum_{m=0}^{N-1} \chi_{N}(m) e^{-i m \theta}\right|^{2}, \quad-\pi \leq \theta \leq \pi .
$$

For $k=0, \pm 1, \pm 2, \ldots$, the moments $\mu_{k}^{(N)}$ with respect to $\psi_{N}(\theta)$ can be computed by the auto-correlation coefficient formulas

$$
\mu_{k}^{(N)}=\frac{1}{2 \pi} \int_{-\pi}^{\pi} e^{-i k \theta} d \psi_{N}(\theta)=\sum_{m=0}^{N-1} \chi_{N}(m) \chi_{N}(m+k) .
$$

Since the trigonometric moment problem for $\left\{\mu_{k}^{(N)}\right\}_{-\infty}^{\infty}$ has a solution 
$\psi_{N}(\theta)$ (Theorem 3.1), there exists a corresponding PPC-fraction

$$
\begin{aligned}
& \delta_{0}^{(N)}-\frac{2 \delta_{0}^{(N)}}{1}+\frac{1}{\overline{\delta_{1}^{(N)} z}}+\frac{\left(1-\left|\delta_{1}^{(N)}\right|^{2}\right) z}{\delta_{1}^{(N)}}+\frac{1}{\overline{\delta_{2}^{(N)}} z} \\
& +\frac{\left(1-\left|\delta_{2}^{(N)}\right|^{2}\right) z}{\delta_{2}^{(N)}}+\cdots,
\end{aligned}
$$

where

$$
\delta_{0}^{(N)}>0, \quad\left|\delta_{k}^{(N)}\right|<1, \quad \delta_{k}^{(N)} \in \mathbb{C}, \quad n=1,2,3, \ldots,
$$

$$
\delta_{k}^{(N)}=\frac{(-1)^{N}}{T_{k}^{(0)}(N)}\left|\begin{array}{cccc}
\mu_{-1}^{(N)} & \mu_{0}^{(N)} & \cdots & \mu_{k-2}^{(N)} \\
\mu_{-2}^{(N)} & \mu_{-1}^{(N)} & \cdots & \mu_{k-3}^{(N)} \\
\vdots & \vdots & & \vdots \\
\mu_{-k}^{(N)} & \mu_{-k+1}^{(N)} & \cdots & \mu_{-1}^{(N)}
\end{array}\right|, \quad k=1,2,3, \ldots
$$

(7.8d) $\quad T_{k}^{(0)}(N)=\left|\begin{array}{cccc}\mu_{0}^{(N)} & \mu_{-1}^{(N)} & \cdots & \mu_{-k+1}^{(N)} \\ \mu_{1}^{(N)} & \mu_{0}^{(N)} & \cdots & \mu_{-k+2}^{(N)} \\ \vdots & \vdots & & \vdots \\ \mu_{k-1}^{(N)} & \mu_{k-2}^{(N)} & \cdots & \mu_{0}^{(N)}\end{array}\right|, \quad k=1,2,3, \ldots$

We let $P_{n}\left(\psi_{N} ; z\right)$ and $Q_{n}\left(\psi_{N} ; z\right)$ denote the $n$th numerator and denominator of the PPC fraction (7.8a). Then $\rho_{n}\left(\psi_{N} ; z\right)=Q_{2 n+1}\left(\psi_{N} ; z\right)$ and $\rho_{n}^{*}\left(\psi_{N} ; z\right)=Q_{2 n}\left(\psi_{N} ; z\right)$ are the $n$th Szegö polynomial and reciprocal polynomial with respect to $\psi_{N}(\theta)$, and they satisfy the recurrence relations

$$
\rho_{0}\left(\psi_{N} ; z\right)=\rho_{0}^{*}\left(\psi_{N} ; z\right)=1
$$

$$
\rho_{n}\left(\psi_{N} ; z\right)=z \rho_{n-1}\left(\psi_{N} ; z\right)+\delta_{n}^{(N)} \rho_{n-1}^{*}\left(\psi_{N} ; z\right), \quad n=1,2,3, \ldots
$$

$(7.9 \mathrm{c}) \rho_{n}^{*}\left(\psi_{N} ; z\right)=\overline{\delta_{n}^{(N)}} z \rho_{n-1}\left(\psi_{N} ; z\right)+\rho_{n-1}^{*}\left(\psi_{N} ; z\right), \quad n=1,2,3, \ldots$

It was shown in [29] that, with $M \geq 1$ fixed, the distribution function $\psi_{N}(\theta)$ converges in the weak star topology, as $N \rightarrow \infty$, to 
the step function $\psi(\theta)$, that is,

$$
\begin{aligned}
\lim _{N \rightarrow \infty} \frac{1}{2 \pi N} \int_{-\pi}^{\pi} h\left(e^{i \theta}\right) d \psi_{N}(\theta) & =\frac{1}{2 \pi} \int_{-\pi}^{\pi} h\left(e^{i \theta}\right) d \psi(\theta) \\
& =\sum_{j=-I}^{I}\left|\alpha_{j}\right|^{2} h\left(e^{i \omega_{j}}\right),
\end{aligned}
$$

for every function $h(z)$ continuous on the unit circle $|z|=1$. Thus, it is not surprising that, with fixed $n \geq M$, the zeros of $\rho_{n}\left(\psi_{N} ; z\right)$ of greatest moduli converge, as $N \rightarrow \infty$, to the critical points $e^{i \omega_{j}}$ on the unit circle. A proof of this result (Theorem 7.1) was given in two separate papers $[\mathbf{3 5}, \mathbf{5 8}]$.

Let

$$
\Delta= \begin{cases}{[ \pm 1, \pm 2, \ldots, \pm I]} & \text { if } \alpha_{0}=0 \\ {[0, \pm 1, \pm 2, \ldots, \pm I]} & \text { if } \alpha_{0}>0\end{cases}
$$

Theorem 7.1. Let $\left\{\chi_{N}(m)\right\}_{m=-\infty}^{\infty}$ be an $N$-terminating signal of the form (1.25). Let $n$ be a fixed integer such that

$$
n \geq M:=2 I+L, \quad L= \begin{cases}0 & \text { if } \alpha_{0}=0 \\ 1 & \text { if } \alpha_{0}>0\end{cases}
$$

Then

(A) For each $N \geq M$, there exist $M$ zeros $z_{j}(n, N)$ of $\rho_{n}\left(\psi_{N} ; z\right)$ that satisfy

$$
\lim _{N \rightarrow \infty} z_{j}(n, N)=e^{i \omega_{j}}, \quad j \in \Delta .
$$

(B) There exists a constant $\kappa_{n}$ such that $0<\kappa_{n}<1$ and the remaining $n-M$ zeros of $\rho_{n}\left(\psi_{N} ; z\right)$ satisfy

$$
\left|z_{j}(n, N)\right| \leq \kappa_{n}<1, \quad \text { for all } N \geq M
$$

It follows that $M$ of the zeros of $\rho_{n}\left(\psi_{N} ; z\right)$ converge to the critical points $e^{i \omega_{j}}$ as $N \rightarrow \infty$, while the remaining (uninteresting) zeros are bounded away from the unit circle. Hence, for each $N \geq M$, it suffices to use the $M$ zeros of $\rho_{n}\left(\psi_{N} ; z\right)$ with greatest moduli as approximations 
of the critical points. We note that, if $n>M$, then $\lim _{N \rightarrow \infty} \rho_{n}\left(\psi_{N} ; z\right)$ may not exist [58].

Our proof of Theorem 7.1 makes use of the following three lemmas.

Lemma 7.2. For each $m=0, \pm 1, \pm 2, \ldots$,

$$
\frac{1}{N} \mu_{m}^{(N)}=\mu_{m}+O\left(\frac{1}{N}\right), \quad \text { as } N \rightarrow \infty .
$$

Proof. Applying equation (1.25) in equation (7.7) yields

$$
\mu_{m}^{(N)}=\sum_{k=0}^{N-m-1}\left[\sum_{j=-I}^{I} \sum_{n=-I}^{I} \alpha_{j} \alpha_{n}^{i\left(\omega_{j}+\omega_{n}\right) k} e^{i m \omega_{n}}\right] .
$$

By summing over $k$ in equation (7.15), the terms with fixed $j$ and $n$ such that $\omega_{j} \neq-\omega_{n}$ yield geometric series that can be expressed as

$$
A_{j, n}(m, N)=\alpha_{j} \alpha_{n} e^{i\left(\omega_{j}+\omega_{n}\right)(N-1) / 2} \frac{\sin \left[\frac{1}{2}\left(\omega_{j}+\omega_{n}\right)(N-m)\right]}{\sin \left[\frac{1}{2}\left(\omega_{j}+\omega_{n}\right)\right]} .
$$

Since the sine term in the denominator does not vanish, there exists a number $A$, independent of $j, n, m$ and $N$ such that

$$
\sup \left[A_{j, n}(m, N): \omega_{j}+\omega_{n} \neq 0,-I \leq j, n \leq I, m \in \mathbb{Z}, N \geq 1\right] \leq A .
$$

The sum of all of the other terms in equation (7.15) (i.e., those with $\left.\omega_{j}+\omega_{n}=0\right)$ is given by $(N-m) \mu_{m}$. Combining these results yields equation (7.14).

Lemma 7.3. (A) For $n=1,2, \ldots, M$,

$$
\delta_{0}^{(N)}=N \delta_{0}+O(1)
$$

and

$$
\delta_{n}^{(N)}=\delta_{n}+O(1 / N), \quad \text { as } N \rightarrow \infty .
$$

(B) For $n=0,1, \ldots, 2 M+1$,

$$
\lim _{N \rightarrow \infty} P_{n}\left(\psi_{N} ; z\right)=P_{n}(z)
$$


and

$$
\lim _{N \rightarrow \infty} Q_{n}\left(\psi_{N} ; z\right)=Q_{n}(z)
$$

convergence being uniform on compact subsets of $\mathbb{C}$.

(C)

$$
\begin{aligned}
\lim _{N \rightarrow \infty} \rho_{M}\left(\psi_{N} ; z\right) & =\lim _{N \rightarrow \infty} Q_{2 M+1}\left(\psi_{N} ; z\right) \\
& =(z-1)^{L} \prod_{j=1}^{I}\left(z-e^{i \omega_{j}}\right)\left(z-e^{-i \omega_{j}}\right),
\end{aligned}
$$

convergence being uniform on compact subsets of $\mathbb{C}$.

(D) If the zeros $z_{j}(M, N)$ of $\rho_{M}\left(\psi_{N} ; z\right)$ are appropriately ordered, then

$$
\begin{aligned}
& \lim _{N \rightarrow \infty} z_{j}(M, N)=e^{i \omega_{j}}, \quad j= \pm 1, \pm 2, \ldots, \pm I, \\
& \left.\lim _{N \rightarrow \infty} z_{0}(M, N)=1 \quad \text { if } L \neq 0 \quad \text { (i.e., } \alpha_{0}>0\right) .
\end{aligned}
$$

Proof.

(A) As $N \rightarrow \infty$, we have by equation (7.14),

$$
\delta_{0}^{(N)}=\mu_{0}^{(N)}=N \mu_{0}+O(1)=N \delta_{0}+O(1),
$$

and by equations $(5.7),(7.8 \mathrm{c})$ and $(7.8 \mathrm{~d})$,

$$
\begin{aligned}
& (-1)^{n}\left|\begin{array}{cccc}
\mu_{-1} & \mu_{0} & \cdots & \mu_{n-2} \\
\mu_{-2} & \mu_{-1} & \cdots & \mu_{n-3} \\
\vdots & \vdots & & \vdots \\
\mu_{-n} & \mu_{-n+1} & \cdots & \mu_{-1}
\end{array}\right|+O\left(\frac{1}{N}\right) \\
\delta_{n}^{(N)} & =\frac{T_{n}^{(0)}+O\left(\frac{1}{N}\right)}{=} \delta_{n}+O\left(\frac{1}{N}\right) .
\end{aligned}
$$

(B) It follows from the difference equations of the form (1.10) satisfied by $P_{n}\left(\psi_{N} ; z\right)$ and $Q_{n}\left(\psi_{N} ; z\right)$ and the analogous equations satisfied by $P_{n}(z)$ and $Q_{n}(z)$ that the coefficients of individual powers of $z$ in $P_{n}\left(\psi_{N} ; z\right)$ and $Q_{n}\left(\psi_{N} ; z\right)$ (or $P_{n}(z)$ 
and $\left.Q_{n}(z)\right)$ are continuous functions of the reflection coefficients $\delta_{k}^{(N)}\left(\right.$ or $\left.\delta_{k}\right), k=0,1, \ldots, n$. Therefore, by equation (7.18), the coefficients in $P_{n}\left(\psi_{N} ; z\right)$ and $Q_{n}\left(\psi_{N} ; z\right)$ converge as $N \rightarrow \infty$ to the corresponding coefficients in $P_{n}(z)$ and $Q_{n}(z)$ for $n=0,1, \ldots, 2 M+1$. Assertion (B) follows from this.

(C) Part (C) is a consequence of (B).

(D) Part (D) is implied by Hurwitz's theorem [25, Theorem 14.3.4] and $(\mathrm{C})$.

Lemma 7.4. Let $\left\{N_{k}\right\}_{k=1}^{\infty}$ be an arbitrary given sequence of natural numbers. Then there exists a subsequence $\left\{N_{k_{\nu}}\right\}_{\nu=1}^{\infty}$ with the following properties:

(A) For $m=1,2,3, \ldots$ and $z \in \mathbb{C}$, the limits

$$
\lim _{\nu \rightarrow \infty} \frac{1}{N_{k_{\nu}}} P_{2 M+m}\left(\psi_{N_{k_{\nu}}} ; z\right)=: P_{2 M+m}\left(\left\{N_{k_{\nu}}\right\}, z\right)
$$

and

$$
\lim _{\nu \rightarrow \infty} Q_{2 M+m}\left(\psi_{N_{k_{\nu}}} ; z\right)=: Q_{2 M+m}\left(\left\{N_{k_{\nu}}\right\}, z\right)
$$

exist, the convergence being uniform on compact subsets of $\mathbb{C}$.

(B) There exists a sequence of polynomials $\left\{U_{m}\left(\left\{N_{k_{\nu}}\right\}, z\right)\right\}_{m=1}^{\infty}$ such that

(7.25a) $P_{2 M+m}\left(\left\{N_{k_{\nu}}\right\}, z\right)=U_{m}\left(\left\{N_{k_{\nu}}\right\}, z\right) P_{2 M}(z), \quad m=1,2,3, \ldots ，$

$Q_{2 M+m}\left(\left\{N_{k_{\nu}}\right\}, z\right)=U_{m}\left(\left\{N_{k_{\nu}}\right\}, z\right) Q_{2 M}(z), \quad m=1,2,3, \ldots$.

(C) For $m=0,1,2, \ldots$,

(7.26) $\lim _{\nu \rightarrow \infty} Q_{2 M+m}\left(\psi_{N_{k_{\nu}}} ; z\right)$

$$
=U_{m}\left(\left\{N_{k_{\nu}}\right\}, z\right)(z-1)^{L} \prod_{j=1}^{I}\left(z-e^{i \omega_{j}}\right)\left(z-e^{-i \omega_{j}}\right),
$$

the convergence being uniform on compact subsets of $\mathbb{C}$.

(D) For $n \geq M$ and $\nu \geq 1$, there exist zeros $z_{j}\left(n, N_{k_{\nu}}\right)$ of $\rho_{n}\left(\psi_{N_{k_{\nu}}} ; z\right)$ such that

$$
\lim _{\nu \rightarrow \infty} z_{j}\left(n, N_{k_{\nu}}\right)=e^{i \omega_{j}}, \quad j= \pm 1, \pm 2, \ldots, \pm I
$$


and

$$
\left.\lim _{\nu \rightarrow \infty} z_{0}\left(n, N_{k_{\nu}}\right)=1 \quad \text { if } \quad L \neq 0 \text {, (i.e., } \alpha_{0}>0\right) \text {. }
$$

Proof.

(A) Since $\left|\delta_{n}^{(N)}\right|<1$, for all $n \geq 1$ and $N \geq 1$, the Cantor diagonalization process insures the existence of a subsequence $\left\{N_{k_{\nu}}\right\}_{\nu=1}^{\infty}$ of $\left\{N_{k}\right\}_{k=1}^{\infty}$ with the property that, for $n=1,2,3, \ldots,\left\{\delta_{n}^{\left(N_{k_{\nu}}\right)}\right\}_{\nu=1}^{\infty}$ is a convergent sequence. We write

$$
\delta_{0}\left(\left\{N_{k_{\nu}}\right\}\right):=\lim _{\nu \rightarrow \infty} \frac{\delta_{0}^{\left(N_{k_{\nu}}\right)}}{N_{k_{\nu}}}=\delta_{0}
$$

and

$$
\delta_{n}\left(\left\{N_{k_{\nu}}\right\}\right):=\lim _{\nu \rightarrow \infty} \delta_{n}^{\left(N_{k_{\nu}}\right)}, \quad n=1,2,3, \ldots
$$

Part (A) follows from this and the fact that, in the polynomials,

$$
\frac{1}{N_{k_{\nu}}} P_{2 M+m}\left(\psi_{N_{k_{\nu}}} ; z\right) \text { and } \quad Q_{2 M+m}\left(\psi_{N_{k_{\nu}}} ; z\right)
$$

the coefficients of individual powers of $z$ are continuous functions of the reflection coefficients $\delta_{n}^{\left(N_{k_{\nu}}\right)}$.

(B) It follows from the difference equations (1.10) that there exist polynomials in $z$ of degree at most $m$ denoted for $\lambda=0,1$ and $m=1,2,3, \ldots$ by

$$
U_{2(M+m)+\lambda}\left(\psi_{N} ; z\right) \text { and } V_{2(M+m)+\lambda}\left(\psi_{N} ; z\right)
$$

such that, for $m \geq 1$ and $\lambda=0,1$,

$$
\begin{aligned}
& Q_{2(M+m)+\lambda}\left(\psi_{N} ; z\right)=U_{2(M+m)+\lambda}\left(\psi_{N} ; z\right) Q_{2 M}\left(\psi_{N} ; z\right) \\
& +\left(1-\left|\delta_{M}^{(N)}\right|^{2}\right) z V_{2(M+m)+\lambda}\left(\psi_{N} ; z\right) Q_{2 M-1}\left(\psi_{N} ; z\right) .
\end{aligned}
$$

The coefficients of individual powers of $z$ in polynomials (7.29) are continuous functions of $\delta_{M}^{(N)}, \delta_{M+1}^{(N)}, \ldots, \delta_{M+m}^{(N)}$. Therefore, for $m \geq 1$ and $\lambda=0,1$, the limits

$$
\lim _{\nu \rightarrow \infty} U_{2(M+m)+\lambda}\left(\psi_{N_{k_{\nu}}} ; z\right)=: U_{2 m+\lambda}\left(\left\{N_{k_{\nu}}\right\}, z\right)
$$


and

$$
\lim _{\nu \rightarrow \infty} V_{2(M+m)+\lambda}\left(\psi_{N_{k_{\nu}}} ; z\right)=: V_{2 m+\lambda}\left(\left\{N_{k_{\nu}}\right\}, z\right)
$$

exist and are polynomials in $z$ of degree at most $m$. Assertion (7.25b) follows from Lemma $7.4(\mathrm{~A}),\left|\delta_{M}\right|=0$, equations (7.30) and (7.31). An analogous argument holds for equation (7.25a).

(C) Assertion (C) follows from equation (7.20) and the fact that $\rho_{M}(z)=Q_{2 M+1}(z)$.

(D) Part (D) is a consequence of (C) and Hurwitz's theorem [25, Theorem 14.3.4].

\section{Proof of Theorem 7.1.}

(A) For a proof by contradiction, we assume that there exists an integer $M_{1}>M$, one of the frequency points $e^{i \omega_{\gamma}}$ where $\gamma \in[0, \pm 1, \pm 2, \ldots, \pm I]$, and a number $\epsilon>0$ such that, for every zero $z_{j}\left(n_{\gamma} ; N_{k}\right)$ of $\rho_{n_{\gamma}}\left(\psi_{N_{k}} ; z\right)$,

$$
\left|z_{j}\left(n_{\gamma}, N_{k}\right)-e^{i \omega_{\gamma}}\right| \geq \epsilon \quad \text { for all } k=1,2,3, \ldots
$$

This is contradicted by Lemma 7.4, and so assertion (A) is valid.

(B) Our proof of (B) is also by contradiction. Let $n$ be a fixed positive integer greater than or equal to $M$, and let $B(n, N)$ denote the set of $(n-M)$ zeros $z_{j}(n, N)$ of $\rho_{n}\left(\psi_{N} ; z\right)$ not considered in Theorem 7.1 (A). Let

$$
B(n):=\bigcup_{N=M}^{\infty} B(n, N) .
$$

Assume that there exists a subsequence $\left\{N_{m}\right\}_{m=M}^{\infty}$ of $\left\{N_{m}\right\}_{m}^{\infty}$ and, for each $m \geq M$, there exists a zero $z_{j}\left(n, N_{m}\right) \in B(n)$ such that

$$
\left|z_{j}\left(n, N_{m}\right)\right| \geq \frac{m-1}{m}, \quad m=M, M+1, M+2, \ldots .
$$

Then, by Lemma 7.4 , there exist a subsequence $\left\{N_{m_{\nu}}\right\}_{\nu=1}^{\infty}$ of $\left\{N_{m}\right\}$ and a polynomial $U\left(\left\{N_{m_{\nu}}\right\}, z\right)$ of degree $n-M$ such that

$$
\lim _{\nu \rightarrow \infty} \rho_{n}\left(\psi_{N_{m_{\nu}}} ; z\right)=U\left(\left\{N_{m_{\nu}}\right\}, z\right) \rho_{M}(z)
$$


the convergence being uniform on compact subsets of $\mathbb{C}$. By a result due to Pan and Saff [58], the $(n-M)$ zeros of $U\left(\left\{N_{m_{\nu}}\right\}, z\right)$ all lie inside the open disk $|z|<1$. Hence, by Hurwitz's theorem [25, Theorem 14.3.4], the zeros $z_{j}\left(n, N_{m_{\nu}}\right)$ of $\rho_{n}\left(\psi_{N_{m_{\nu}}} ; z\right)$ are contained inside a disk of the form $[z \in \mathbb{C}$ : $|z|<k<1]$, that is, the zeros $z_{j}\left(n, N_{m_{\nu}}\right)$ are bounded away from the unit circle $|z|=1$. This contradicts conditions (7.33).

8. Wiener-Levinson filters. Wiener-Levinson filters are intimately related to Szegö polynomials and PPC-fractions. Let $l$ denote the linear space of doubly infinite sequences of real numbers $x=\{x(m)\}_{-\infty}^{\infty}$ (called signals) over the field $\mathbb{R}$. A linear map $T$ of $l$ into $l$ is called a digital filter if $T$ is shift-invariant, that is,

$$
T S=S T
$$

where the unit shift operator $S$ is defined by

$$
(S x)(m)=x(m-1), \quad m=0, \pm 1, \pm 2, \ldots, \quad x \in l .
$$

We restrict our consideration to signals of the form (1.25) and let $x_{N}=\left\{x_{N}(m)\right\}_{-\infty}^{\infty}$, where

$$
x_{N}(m)= \begin{cases}B\left(t_{m}\right) & m=0,1,2, \ldots, N-1 \\ 0 & \text { if } m<0 \text { or } m \geq N .\end{cases}
$$

Let $T$ be a linear map of the form $y_{N}=T x_{N}$, where

$$
y_{N}(m)= \begin{cases}-\sum_{j=1}^{n} g_{j}^{(n)} x_{N}(m-j) & \text { for } m \geq 1, g_{j}^{(n)} \in \mathbb{R}, \\ 0 & \text { for } m \leq 0,\end{cases}
$$

for $n<N$. It can readily be shown that $T$ is a digital filter. The output $y_{N}$ of the filter is said to be a "linear prediction" of the input $x_{N}$ since each element $y_{N}(m)$ is a linear combination of the preceding elements $x_{N}(m-1), x_{N}(m-2), \ldots, x_{N}(0)$. The filter $T$ is a Wiener-Levinson filter if the coefficients $g_{j}^{(n)}$ are chosen in such a manner as to minimize 
the sum of squares of the residuals

$$
\begin{gathered}
\epsilon_{N}^{(n)}(m)=x_{N}(m)-y_{N}(m)=\sum_{j=0}^{n} g_{j}^{(n)} x_{N}(m-j) \\
m=0, \pm 1, \pm 2, \ldots
\end{gathered}
$$

where $g_{0}^{(n)}=1$. It follows from equation (7.7) that

$$
\begin{aligned}
\sum_{m=-\infty}^{\infty}\left[\epsilon_{N}^{(n)}(m)\right]^{2} & =\sum_{j=0}^{n} \sum_{k=0}^{n} g_{j}^{(n)} g_{k}^{(n)} x_{N}(m-j) x_{N}(m-k) \\
& =\sum_{j=0}^{n} \sum_{k=0}^{n} g_{j}^{(n)} g_{k}^{(n)} \mu_{j-k}^{(N)} \\
& =\frac{1}{2 \pi} \int_{-\pi}^{\pi}\left|\sum_{j=0}^{n} g_{j}^{(n)} e^{-i j \theta}\right|^{2} d \psi_{N}(\theta) \\
& =\left\langle G_{n}, G_{n}\right\rangle_{\psi_{N}},
\end{aligned}
$$

where

$$
G_{n}(z)=\sum_{j=0}^{n} g_{j}^{(n)} z^{-j}
$$

Since $z^{n} G_{n}(z)$ is a polynomial in $z$ of degree $n$, it follows from the extremal property of Szegö polynomials (Theorem 4.2) that

$$
\begin{aligned}
\min _{g_{j}^{(n)} \in \mathbb{R}} \sum_{m=-\infty}^{\infty}\left[\epsilon_{N}^{(n)}(m)\right]^{2} & =\min _{g_{j}^{(n)} \in \mathbb{R}}\left\langle z^{n} G_{n}, z^{n} G_{n}\right\rangle_{\psi_{N}} \\
& =\left\langle\rho_{n}, \rho_{n}\right\rangle_{\psi_{N}}
\end{aligned}
$$

where $\rho_{n}\left(\psi_{N} ; z\right)$ is the monic, $n$th degree Szegö polynomial with respect to $\psi_{N}$ defined by equation (7.6). Therefore, the frequency response $G_{n}\left(e^{i \theta}\right)$ of the Wiener-Levinson filter is given by

$$
G_{n}\left(e^{i \theta}\right)=e^{-i n \theta} \rho_{n}\left(\psi_{N} ; e^{i \theta}\right) .
$$

9. Illustrations from computational experiments. The theory and methods for frequency analysis described in the previous sections are illustrated here by two examples which first appeared in [38]. 
For both examples, the input discrete time signals $\chi_{N}(m)$ in equation (1.25a) are defined with $I=4$. Values of $\alpha_{j}$ and $\omega_{j}$ used in these examples are given as follows:

Example 9.1.

\begin{tabular}{|c|c|c|c|c|c|}
\hline$j$ & 0 & 1 & 2 & 3 & 4 \\
\hline $2 \alpha_{j}$ & 0 & 1 & 1 & 1 & 1 \\
\hline$\omega_{j}$ & 0 & $\pi / 6$ & $\pi / 3$ & $\pi / 2$ & $3 \pi / 4$ \\
\hline
\end{tabular}

Example 9.2.

\begin{tabular}{|c|c|c|c|c|c|}
\hline$j$ & 0 & 1 & 2 & 3 & 4 \\
\hline $2 \alpha_{j}$ & 0 & 1 & 1 & 1 & 10 \\
\hline$\omega_{j}$ & 0 & $\pi / 6$ & $\pi / 3$ & $\pi / 2$ & $3 \pi / 4$ \\
\hline
\end{tabular}

For each example, the Szegö polynomials $\rho_{k}\left(\psi_{N}, z\right)$ for several values of the degree $k$ and sample size $N$ have been computed using the Levinson algorithm (4.10). The zeros $z_{j}(k, z)$ of $\rho_{k}\left(\psi_{N}, z\right)$ have also been computed.

Figures 1 and 4 are graphs of $\left|z_{j}(k, N)-e^{i \omega_{j}}\right| \operatorname{versus} N$ in a logto-log scale for six representative values of $k$ and for each of the four frequencies $\omega_{j}$. In all cases, the zero $z_{j}(k, N)$ nearest to a critical point $e^{i \omega_{j}}$ was chosen, and we see that $\left|z_{j}(k, N)-e^{i \omega_{j}}\right|$ appears to approach zero as $N$ tends to infinity on the order of $O(1 / N)$ as expected from Lemma 7.3. The graphs in Figures 2, 3, 5 and 6 show zeros $z_{j}(k, N)$ as endpoints of lines radiating from the origin with $N=101$ for Figures 2 and 3 and $N=401$ for Figures 5 and 6 . The trend in all of the graphical illustrations is that the zeros $z_{j}(k, N)$ appear to converge to corresponding critical points $e^{i \omega_{j}}$ as $k$ increases and also as $N$ tends to infinity. It is of interest to note that, for fixed $k$, the "uninteresting zeros" $z_{j}(k, N)$ that are bounded away from the unit circle as $N$ increases (Theorem 7.1 (B)) appear to be uniformly distributed around the unit circle which is a characteristic that would be expected if they were produced mainly by random white noise. 

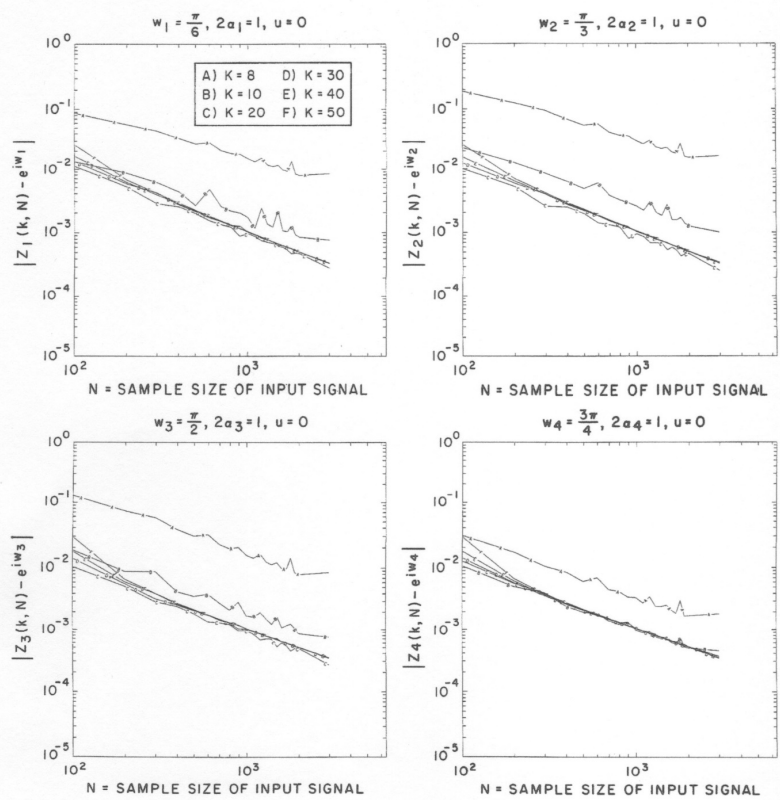

Figure 1. For Example 1, the graphs of $\left|z_{j}(k, N)-e^{i \omega_{j}}\right|$ versus $N$ in a $\log -$ $\log$ scale, where $z_{j}(k, N)$ denotes a zero of the Szegö polynomial $\rho_{k}\left(\psi_{N}, z\right)$, $I=4$. 

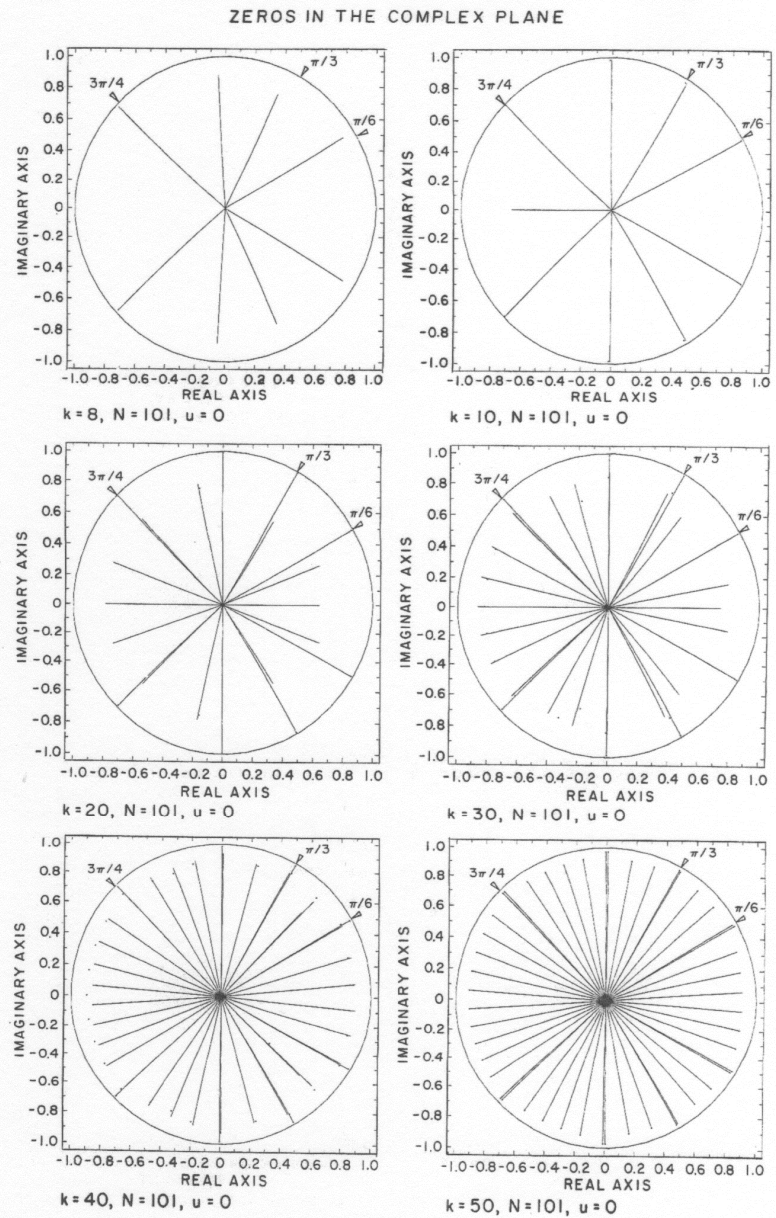

FiguRE 2. For Example 1, zeros $z_{j}(k, N)$ of Szegö polynomial $\rho_{k}\left(\psi_{N}, z\right)$ are shown as endpoints of lines radiating from the origin; $N=101, I=4$. 

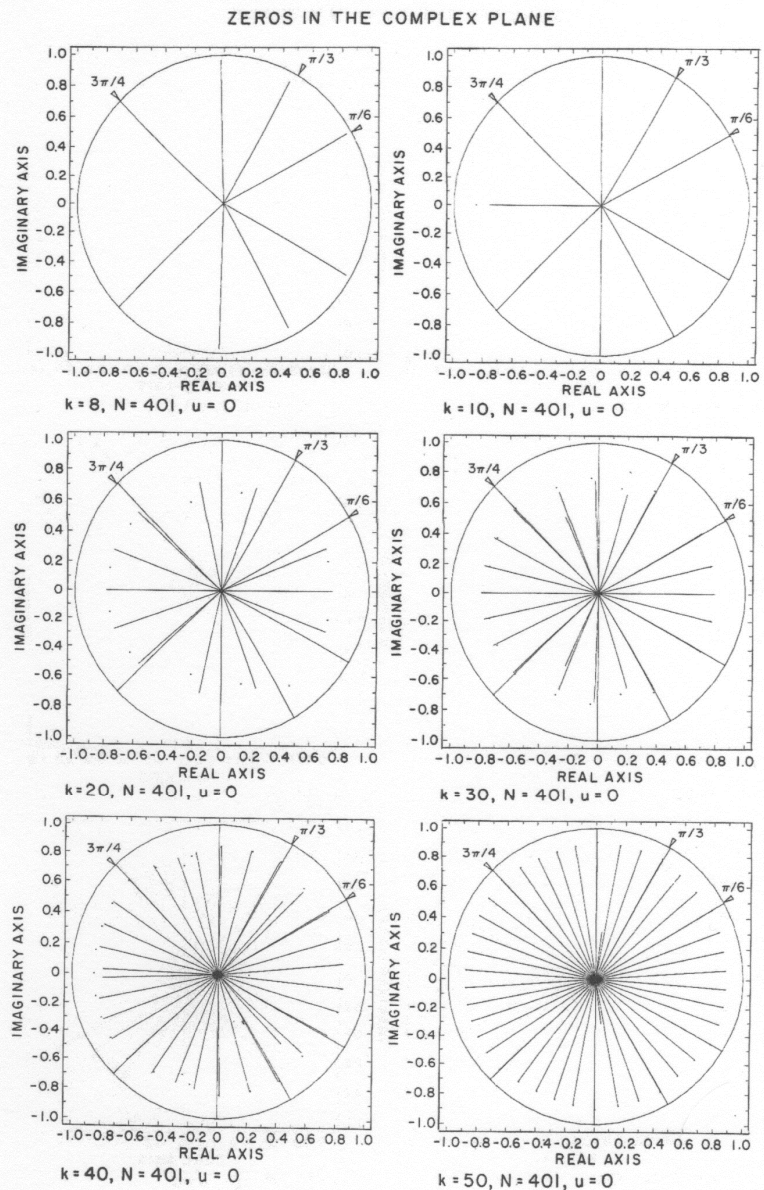

FiguRE 3. For Example 1, zeros $z_{j}(k, N)$ of Szegö polynomial $\rho_{k}\left(\psi_{N}, z\right)$ are shown as endpoints of lines radiating from the origin; $N=401, I=4$. 

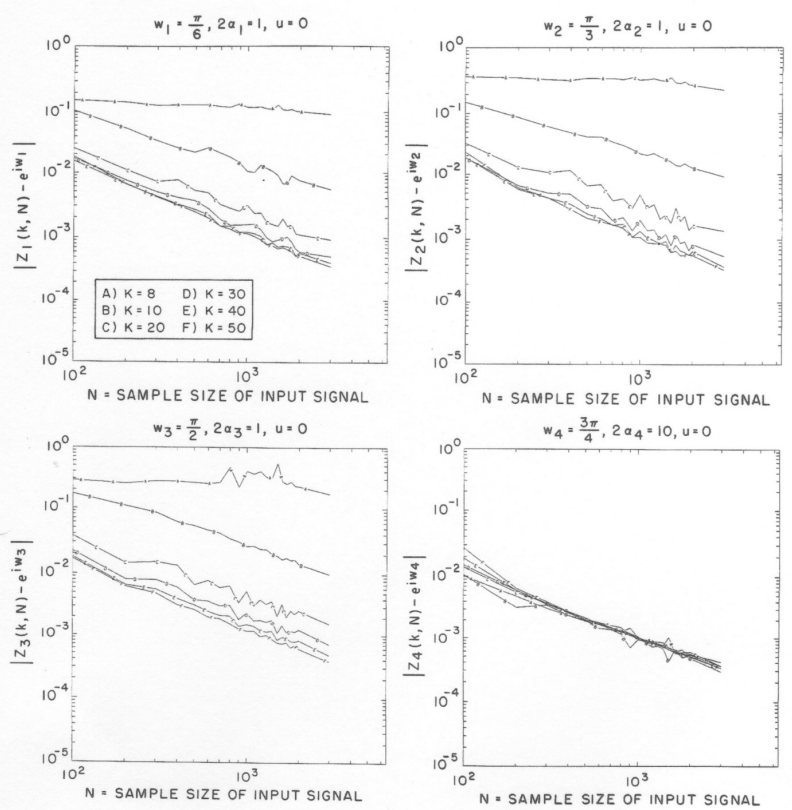

FiguRE 4. For Example 2, the graphs of $\left|z_{j}(k, N)-e^{i \omega_{j}}\right|$ versus $N$ in a $\log -$ $\log$ scale, where $z_{j}(k, N)$ denotes a zero of the Szegö polynomial $\rho_{k}\left(\psi_{N}, z\right)$, $I=4$. 

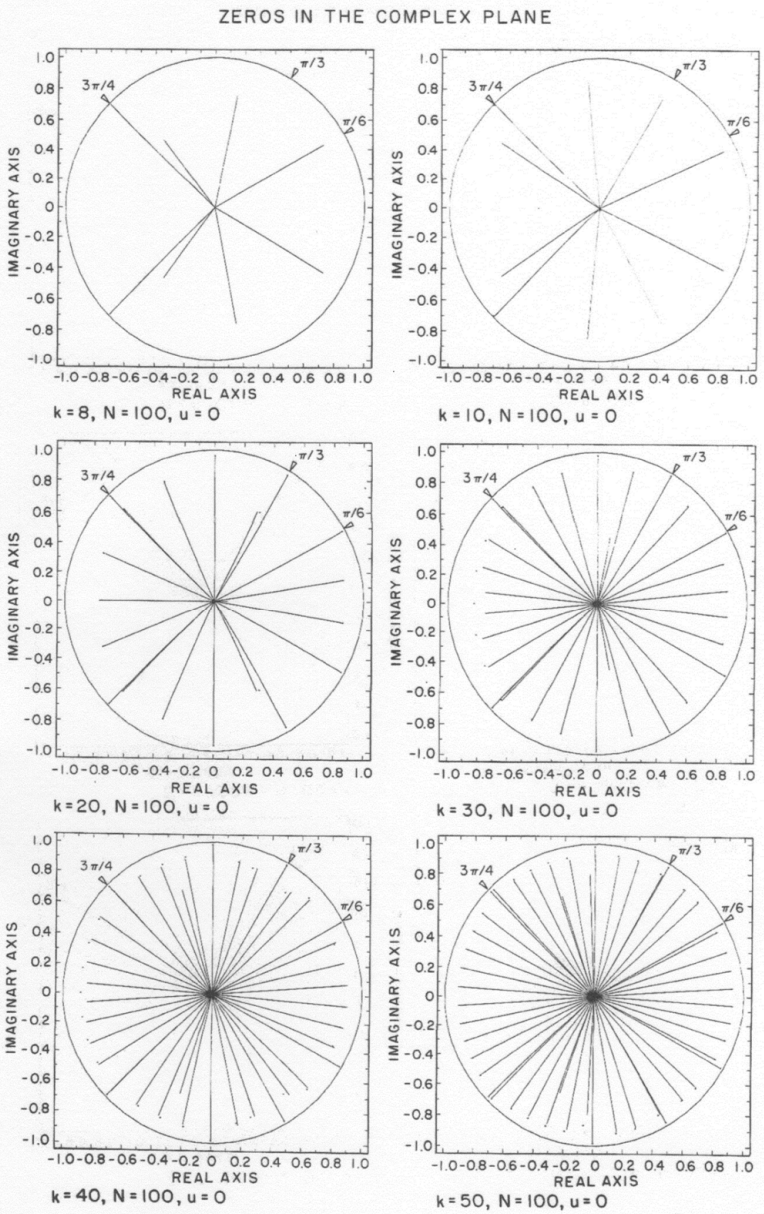

Figure 5. For Example 2, zeros $z_{j}(k, N)$ of Szegö polynomial $\rho_{k}\left(\psi_{N}, z\right)$ are shown as endpoints of lines radiating from the origin, $N=100, I=4$. 

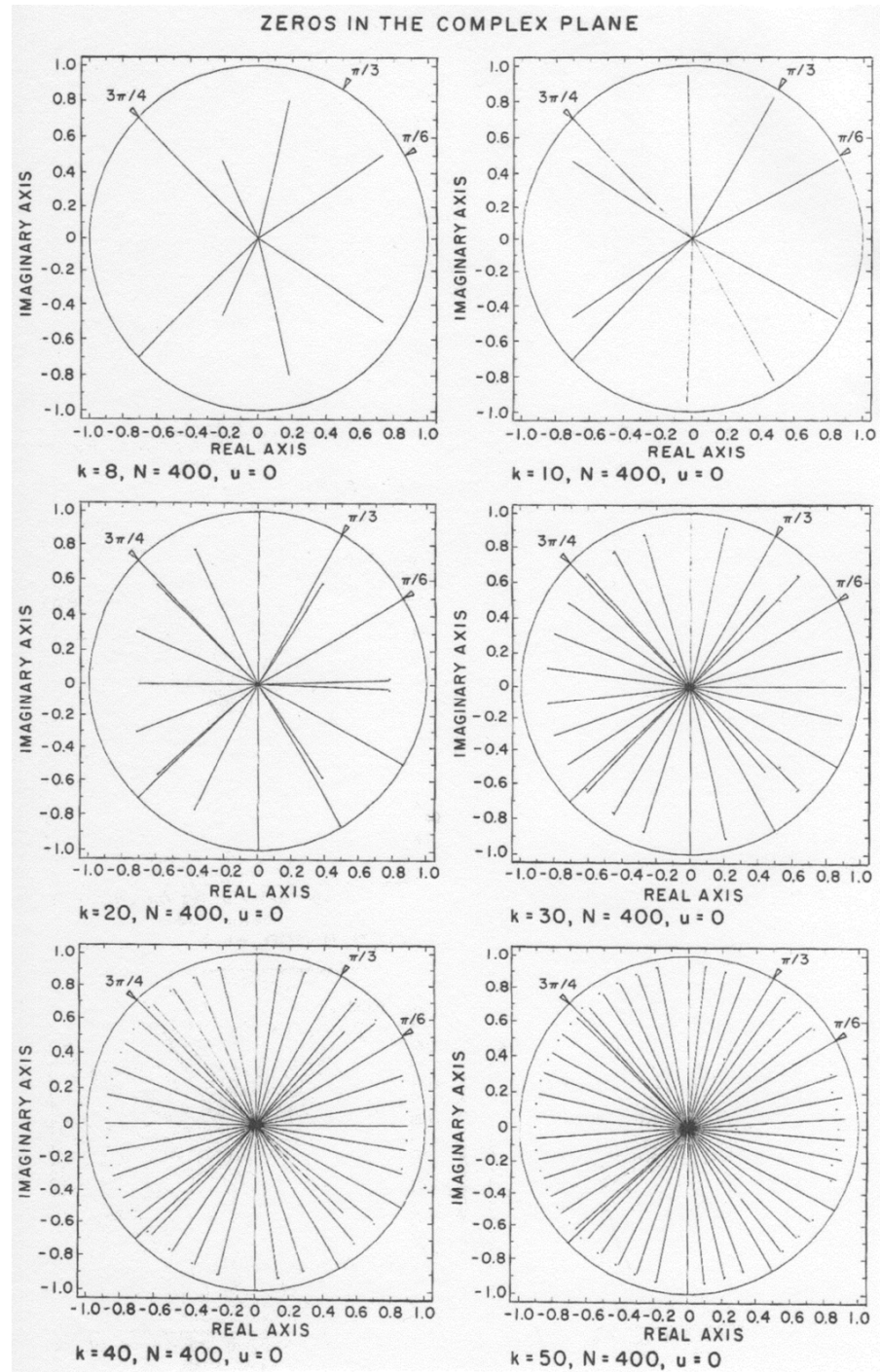

FiguRE 6. For Example 2, zeros $z_{j}(k, N)$ of Szegö polynomial $\rho_{k}\left(\psi_{N}, z\right)$ are shown as endpoints of lines radiating from the origin, $N=400, I=4$. 


\section{REFERENCES}

1. N.I. Akhiezer, The classical moment problem and some related questions in analysis, Hafner, New York, 1965.

2. N.I. Akhiezer and M. Krein, Über Fouriersche Reihen beschränkter summierbarer Funktionen und ein neues Extremumproblem, Comm. Soc. Math. Kharkov 9 (1934), 3-28; 10 (1934), 3-32.

3. G.S. Ammar and W. B. Gragg, Superfast solution of real positive definite Toeplitz systems, SIAM J. Matrix Anal. Appl. 9 (1988), 61-76.

4. B.S. Atal and M.R. Schroeder, Predictive coding of speech signals, Proc. 1967 IEEE Conference on Communication and Processing, 360-361.

5. A. Bultheel, Algorithms to compute the reflection coefficients of digital filters, in Numerische Methoden der Approximationstheorie, L. Collatz, C. Meinardus and H. Werner, eds., Birkheiser, Basel, 1984.

6. R.R. Bitmead and B.D.O. Anderson, Asymptotically fast solution of Toeplitz and related systems of linear equations, Linear Alg. Appl. 34 (1980), 103-116.

7. C. Carathéodory, Über den Variabilitätsbereich der Koeffizienten von Potenzreihen die gegebene Werte nicht annehmen, Math. Ann. 64 (1907), 95-115.

8. , Über den Variabilitätsbereich der Fourierschen Funktionen, Rend. Circ. Mat. Palermo 32 (1911), 193-217.

9. A. Cuyt, V. Brevik Petersen, B. Verdonk, H. Waadeland and W. B. Jones, Handbook of continued fractions for special functions, Springer, New York, 2008.

10. G. Cybenko, The numerical stability of the Levinson-Durbin algorithm for systems of equations, SIAM J. Sci. Stat. Comp. 1 (1980), 303-319.

11. Moment problems and low rank Toeplitz applications, Circuits Syst. Sig. Proc. 1 (1982), 345-366.

12. L. Daruis, O. Njåstad and W. VanAssche, Para-orthogonal polynomials in frequency analysis, Rocky Mountain J. Math. 33 (2003), 629-645.

13. Szegö quadrature and frequency analysis, Electr. Trans. Num. Anal. 19 (2005), 48-57.

14. P. Delsarte and Y. Genin, Spectral properties of finite Toeplitz matrices, in Mathematical theory of networks and systems, Lect. Note Contr. Inform. Sci., P.A. Fuhrmann, ed., Springer, 1984.

15. P. Delsarte, Y. Genin and Y. Kamp, Pseudo-Carathéodory functions and Hermitian Toeplitz matrices, Philips J. Res. 41 (1986), 1-54.

16. P. Delsarte, Y. Genin, Y. Kamp and P. van Dooren, Speech modeling and the trigonometric moment problem, Philips J. Res. 37 (1982), 277-292.

17. G. Freud, Orthogonal polynomials, Pergamon, Oxford, 1971.

18. Ya.L. Geronimus, Polynomials orthogonal on a circle and their applications, Amer. Math. Soc. Trans. 104, 1954.

19. , Orthogonal polynomials, Consultants Bureau, New York, 1961.

20. I. Gohberg, ed., I. Schur methods in operator theory and signal processing, Oper. Th. Adv. Appl. 18, Birkhauser, Basel, 1986. 
21. U. Grenander and G. Szegö, Toeplitz forms and their applications, University of California Press, Berkeley, 1958.

22. H. Hamburger, Über eine Erweiterung des Stieltjesschen Momentenproblems, Parts I, II and III, Math. Ann. 81 (1920), 235-319; 82 (1921), 120-164, $168-187$.

23. P. Henrici, Applied and computational complex analysis, Volume 2 , in Special functions, integral transforms, asymptotics and continued fractions, Wiley-Hill, New York, 1977.

24. Über Potenzreihen mit positivem reellen Teil im Einheitskreis, Ber. Verh. Sachs. Ges. Wiss., Leipzig, Math. Phys. 63 (1911), 501-511.

25. E. Hille, Analytic function theory, Volume II, Ginn and Company, 1962.

26. I.I. Hirschmann, Jr., Recent developments in the theory of finite Toeplitz operators, in Adv. Prob. Rel. Top. 1, P. Ney, ed., Dekker, New York, 1971.

27. K. Hoffman and R. Kunze, Linear algebra, 2nd edition, Prentice Hall, 1971.

28. W.B. Jones and O. Njåstad, Applications of Szegö polynomials to digital signal processing, Rocky Mountain J. Math. 21 (1991), 387-436.

29. W.B. Jones, O. Njåstad and E.B. Saff, Szegö polynomials associated with Wiener-Levinson filters, J. Comp. Appl. Math. 32 (1990), 387-407.

30. W.B. Jones, O. Njåstad and W.J. Thron, Continued fractions and strong Hamburger moment problems, Proc. Lond. Math. Soc. 47 (1983), 363-384.

31. , Orthogonal Laurent polynomials and the strong Hamburger moment problem, J. Math. Anal. Appl. 98 (1984), 528-554.

32. Continued fractions associated with the trigonometric and other strong moment problems, Constr. Approx. 2 (1986), 197-211.

33. _ Schur fractions, Perron-Carathéodory fractions and Szegö polynomials, A survey, Lect. Notes Math. 1199, W.J. Thron, ed., Springer, 1986.

34. , Moment theory, orthogonal polynomials, quadrature and continued fractions associated with the unit circle, Bull. Lond. Math. Soc. 21 (1989), 113-152.

35. W.B. Jones, O. Njåstad, W.J. Thron and H. Waadeland, Szegö polynomials applied to frequency analysis, J. Comp. Appl. Math. 46 (1993), 217-228.

36. W.B. Jones, O. Njåstad and H. Waadeland, Asymptotics for Szegö polynomial zeros, Numer. Alg. 3 (1992), 255-264.

37. Application of Szegö polynomials to frequency analysis, SIAM J. Math. Anal. 25 (1994), 491-512.

38. , Asymptotics of zeros of orthogonal and para-orthogonal polynomials in frequency analysis, in Continued fractions and orthogonal functions: Theory and applications, S. Clement Cooper and W.J. Thron, Marcel Dekker, Inc., New York, 1994.

39. W.B. Jones and V. Petersen, Continued fractions and Szegö polynomials in frequency analysis and related topics, Acta Appl. Math. 61 (2000), 149-174.

40. W.B. Jones, V. Petersen and H. Waadeland, Convergence of PPC-fraction approximants in frequency analysis, Rocky Mountain J. Math. 33 (2003), 525-544. 
41. W.B. Jones and E.B. Saff, Szegö polynomials and frequency analysis, in Approximation theory, G. Anastassiou, ed., Marcel Dekker, Inc., 1992.

42. W.B. Jones and W.J. Thron, Continued fractions: Analytic theory and applications, Encycl. Math. Appl. 11, Addison Wesley, 1980; Cambridge University Press, 1984, digitally printed version, 2008.

43. , Orthogonal Laurent polynomials and Gaussian quadrature, in Quantum mechanics in mathematics, chemistry and physics, K. Gustafson and W.P. Reinhardt, eds., Plenum Press, New York, 1980.

44. , A constructive proof of convergence of the even approximants of positive PC-fractions, Rocky Mountain J. Math. 19 (1989), 199-210.

45. W.B. Jones, W.J. Thron and H. Waadeland, A strong Stieltjes moment problem, Trans. Amer. Math. Soc. 261 (1980), 503-528.

46. H.J. Landau, ed., Moments in mathematics, Proc. Sympos. Appl. Math. 37, American Mathematical Society, Providence, 1987.

47. N. Levinson, The Wiener RMS (root mean square) error criterion in filter design prediction, J. Math. Phys. 25 (1947), 261-278.

48. X. Li, Asymptotics of columns in the table of orthogonal polynomials with varying measures, Meth. Appl. Anal. 2 (1995), 222-236.

49. L. Lorentzen and $\mathrm{H}$. Waadeland, Continued fractions with applications, Stud. Comp. Math. 3, North-Holland, 1992.

50. Continued fractions, Volume 1: Convergence theory, Atlantis Press/World Scientific, Amsterdam, 2008.

51. P.S. Lubinsky, A survey of general orthogonal polynomials on finite and infinite intervals, Acct. Appl. Math. 10 (1987), 237-296.

52. J.D. Markel and A.N. Gray, Jr., Linear prediction of speech, Springer, New York, 1976.

53. H.N. Mhaskar and E.B. Saff, On the distribution of zeros of polynomials orthogonal on the unit circle, J. Approx. Theor. 63 (1990), 30-38.

54. O. Njåstad and W.J. Thron, The theory of sequences of orthogonal Lpolynomials, Det. Kong. Norske Videns. Selskab 1 (1983), 54-91.

55. O. Njåstad and H. Waadeland, Asymptotic properties of zeros of orthogonal rational functions, J. Math. Anal. Appl. 77 (1997), 255-275.

56. , Generalized Szegö theory in frequency analysis, J. Math. Anal. Appl. 206 (1997), 280-307.

57. K. Pan, Asymptotics for Szegö polynomials associated with Wiener-Levinson filters, J. Comp. Appl. Math. 46 (1993), 387-394.

58. K. Pan and E.B. Saff, Asymptotics for zeros of Szegö polynomials associated with trigonometric polynomial signals, J. Approx. Th. 7 (1992), 239-251.

59. O. Perron, Die Lehre von den Kettenbrüchen, Volume 2, Teubner, Stuttgart, 1957.

60. V. Petersen, A theorem on Toeplitz determinants containing Tchebycheff polynomials of the first kind, The Royal Norwegian Soc. Sci. Lett. Trans. 4 (1996). 
61. V. Petersen, Szegö polynomials in frequency analysis: Observations on speed of convergence, Comm. Anal. Th. Cont. Fract. 5 (1996), 27-33.

62. A combination of two methods in frequency analysis: The $R(N)$ process, Lect. Notes Pure Appl. Math. 199, W.B. Jones and A. Sri Ranga, eds., Marcel Dekker, New York, 1998.

63. Zeros of Szegö polynomials used in frequency analysis, Orthogonal functions, moment theory, and continued fractions: Theory and applications, Lect. Notes Pure Appl. Math. 199, W.B. Jones and A. Sri Ranga, eds., Marcel Dekker, New York, 1998.

64. On measures in frequency analysis, J. Compl. Appl. Math. 105 (1999), 437-443.

65. , Weak convergence and boundedness properties of measures in frequency analysis, J. Math. Anal. Appl. 245 (2000), 87-104.

66. L.R. Rabiner and R.W. Schafer, Digital processing of speech signals, Prentice-Hall, Englewood Cliffs, NJ, 1978.

67. F. Riesz, Sur certaines systèmes singuliers d'equations integrales, Ann. Sci. Ecole Norm. Sup. 28 (1911), 33-62.

68. M. Riesz, Sur le problème des moments, Troisième Note, Ark. Mat. Astr. Och Fys. 17 (1923), 1-52.

69. I. Schur, Über Potenzreihen die im Innern des Einheitskreises beschränkt sind, J. reine angew. Math. 147 (1917), 205-232; 148 (1918-19), 122-145.

70. B. Simon, Orthogonal polynomials on the unit circle, Parts 1 and 2, Amer. Math. Soc. Colloq. Pub. 54 (2004), Providence, RI.

71. T.J. Stieltjes, Rechèrches sur les fractions continues, Ann. Fac. Toul. 8 (1894), 1-122; 9 (1894), 1-47; Thomas Jan Stieltjes Oeuvres Completes Collected Papers, Volume II, Gerrit van Dijk, ed., Springer, 1993 (in English).

72. G. Szegö, Orthogonal polynomials, American Mathematical Society, Providence, 1959 .

73. O. Toeplitz, Über die Fouriersche Entwicklung positiver Funktionen, Rend. Circ. Mat. Palermo 32 (1911), 191-192.

74. W.J. Thron, The theory of functions of a complex variable, John Wiley and Sons, New York, 1953.

75. H.S. Wall, Analytic theory of continued fractions, D. Van Nostrand, New York, 1948.

76. N. Wiener, Extrapolation, interpolation and smoothing of stationary time series, published jointly with The Technology Press of MIT and Wiley, Inc., New York, 1949. 
Department of Computer Science, Mathematics and Statistics, Colorado Mesa University, 1100 North Ave, Grand Junction, CO 81501

Email address: cbonan@coloradomesa.edu

Department of Mathematics, Campus Box 395, University of Colorado, Boulder, Colorado, 80309

Email address: william.jones@colorado.edu

Department of Mathematical Sciences, Norwegian University of Science and Technology, 7491 Trondheim, Norway

Email address: njastad@math.ntnu.no 